\title{
HCS Campaign to Identify Selective Inhibitors of IL-6-Induced STAT3 Pathway Activation in Head and Neck Cancer Cell Lines
}

Paul A. Johnston, ${ }^{1-3}$ Malabika Sen ${ }^{4}$ Yun Hua, ${ }^{1}$

Daniel P. Camarco, ${ }^{1}$ Tong Ying Shun, ${ }^{3}$ John S. Lazo, ${ }^{5}$

Gabriela Mustata Wilson, ${ }^{6,7, *}$ Lynn O. Resnick,

Matthew G. LaPorte, ${ }^{6}$ Peter Wipf, ${ }^{1,2,6,8}$

Donna M. Huryn, ${ }^{1,6}$ and Jennifer R. Grandis ${ }^{9}$

${ }^{1}$ Department of Pharmaceutical Sciences, School of Pharmacy, University of Pittsburgh, Pittsburgh, Pennsylvania.

${ }^{2}$ University of Pittsburgh Cancer Institute, Pittsburgh,

Pennsylvania.

${ }^{3}$ Pittsburgh Specialized Application Center, University of Pittsburgh Drug Discovery Institute, Pittsburgh, Pennsylvania. ${ }^{4}$ Department of Otolaryngology, University of Pittsburgh, Pittsburgh, Pennsylvania.

${ }^{5}$ Departments of Pharmacology and Chemistry, University of Virginia, Charlottesville, Virginia.

${ }^{6}$ University of Pittsburgh Chemical Diversity Center, Pittsburgh, Pennsylvania.

${ }^{7}$ Department of Health Services and Health Administration, College of Nursing and Health Professions, University of Southern Indiana, Evansville, Indiana.

${ }^{8}$ Department of Chemistry, University of Pittsburgh, Pittsburgh, Pennsylvania.

${ }^{9}$ Clinical and Translational Science Institute, Otolaryngology Head and Neck Surgery, University of California,

San Francisco, California.

${ }^{*}$ Current address: Department of Health Services and Health Administration, College of Nursing and Health Professions, University of Southern Indiana, Evansville, Indiana.

\section{ABSTRACT}

Signal transducer and activator of transcription factor 3 (STAT3) is hyperactivated in head and neck squamous cell carcinomas (HNSCC). Cumulative evidence indicates that IL-6 production by HNSCC cells and/or stromal cells in the tumor microenvironment activates STAT3 and contributes to tumor progression and drug resistance. A library of 94,491 compounds from the Molecular Library Screening Center Network (MLSCN) was screened for the ability to inhibit interleukin-6 (IL-6)-induced pSTAT3 activation. For contractual reasons, the primary high-content screening (HCS) campaign was conducted over several months in 3 distinct phases; 1,068 (1.1\%) primary HCS actives remained after cytotoxic or fluorescent outliers were eliminated. One thousand one hundred eighty-seven compounds were cherry-picked for confirmation; actives identified in the primary HCS and compounds selected by a structural similarity search of the remaining MLSCN library using hits identified in phases I and II of the screen. Actives were confirmed in PSTAT3 IC $C_{50}$ assays, and an IFNy-induced pSTAT1 activation assay was used to prioritize selective inhibitors of STAT3 activation that would not inhibit STAT1 tumor suppressor functions. Two hundred three concentration-dependent inhibitors of IL-6-induced PSTAT3 activation were identified and 89 of these also produced I $C_{50}$ against IFN- $\gamma$-induced pSTAT1 activation. Forty-nine compounds met our hit criteria: they reproducibly inhibited IL-6-induced PSTAT3 activation by $\geq 70 \%$ at $20 \mu \mathrm{M}$; their pSTAT3 activation $I C_{50}$ s were $\leq 25 \mu M$; they were $\geq 2$-fold selective for PSTAT3 inhibition over pSTAT1 inhibition; a cross target query of PubChem indicated that they were not biologically promiscuous; and they were $\geq 90 \%$ pure. Twenty-six chemically tractable hits that passed filters for nuisance compounds and had acceptable drug-like and ADME-Tox properties by computational evaluation were purchased for characterization. The hit structures were distributed among 5 clusters and 8 singletons. Twenty-four compounds inhibited IL-6-induced pSTAT3 activation with $I C_{50} \mathrm{~S}$ $\leq 20 \mu \mathrm{M}$ and 13 were $\geq 3$-fold selective versus inhibition of pSTAT1 activation. Eighteen hits inhibited the growth of HNSCC cell lines with average $I C_{50} S \leq 20 \mu \mathrm{M}$. Four chemical series were progressed into lead optimization: the guanidinoquinazolines, the triazolothiadiazines, the amino alcohols, and an oxazole-piperazine singleton.

\section{INTRODUCTION}

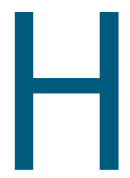

yperactivation of the signal transducer and activator of transcription 3 (STAT3) signaling pathway has been implicated in the development, progression, and maintenance of many cancers. ${ }^{1-5}$ Elevated levels of activated STAT3, phosphorylated at a single tyrosine residue (Y705) of its transactivation domain, are frequently detected in human tumor biopsies and are often associated with poor clinical prognosis. ${ }^{1,2,4,6-8}$ Head and neck squamous 
cell carcinoma (HNSCC) tumors and cell lines exhibit high pSTAT3-Y705 expression levels. ${ }^{4,5,7-11}$ HNSCC is the eighth leading cause of cancer worldwide with an incidence of $\sim 600,000$ new cases and $\sim 300,000$ deaths per annum. ${ }^{12-14}$ Smoking, alcohol use, genetics, and human papillomavirus (HPV) infection contribute to the carcinogenesis and pathology of HNSCC. ${ }^{12-14}$ Despite reductions in cigarette smoking rates, the incidence of HNSCC continues to rise in developed countries, where HPV-associated HNSCC comprises a pathologically distinct group that contributes to the higher prevalence. ${ }^{12-15}$ The heterogeneous genetic nature and pathological diversity of HNSCC have hampered the development of effective drug therapies. ${ }^{12-14}$ Only 6 drugs are currently approved by the FDA for HNSCC treatment, typically in combination with front line surgical resection and radiation therapy: methotrexate and 5-fluorouracil were approved in the 1950s, bleomycin and cisplatin were approved in the 1970s, and docetaxel and cetuximab were approved in 2006. ${ }^{12,13}$ Approximately 10-25\% of HNSCC patients will respond to single-drug treatments, and although response rates may be higher with some drug combinations, they generally do not prolong survival over single-agent therapy. ${ }^{12,13}$ The current surgery, radiation, and chemotherapy regimens have only produced limited improvement in HNSCC prognosis and the 5-year survival rate has been 50\% for over 30 years. ${ }^{12-14,16-18}$ Patients with recurrent or metastatic HNSCC have median survival rates of 6-12 months, ${ }^{12,13,16}$ and there is therefore an urgent need for new and effective therapies.

STAT3 mutations that constitutively activate STAT3 signaling are rare in all cancers, including HNSCC. ${ }^{4}$ An exception are the somatic mutations in the STAT3 SH2 dimerization and activation domain that are observed in $40 \%$ of patients with large granular lymphocytic leukemia, a rare lymphoproliferative disorder characterized by the expansion of clonal $\mathrm{CD}^{+} \mathrm{CD}^{+}$cytotoxic T lymphocytes. ${ }^{19,20}$ The STAT3 $\mathrm{SH} 2$ domain mutations produce a larger hydrophobic protein surface that is associated with increased phosphorylation of STAT3 and nuclear localization. ${ }^{19,20}$ However, other components of the STAT3 signaling pathway that lead to elevated pSTAT3-Y705 levels are more frequently altered in many cancer types; overexpression and amplification of epidermal growth factor receptor (EGFR) levels, EGFR mutations that result in constitutive receptor tyrosine kinase (RTK) activation, overexpression of Src family kinases (SFKs), and mutations that hyperactivate Janus kinases (JAKs). ${ }^{4-7,21-24}$ The monoclonal antibody (mAb) cetuximab, which blocks downstream EGFR signaling by antagonizing ligand binding to EGFRs, was developed and approved to target the high levels of EGFR expression frequently ( $>90 \%$ ) observed in HNSCC. ${ }^{5,7,10,25}$ Despite ubiquitous overexpression of EGFR in HNSCC, most patients fail to respond to EGFR inhibitor treatment. ${ }^{5,7,10,25}$ Several mechanisms of HNSCC resistance to EGFR-targeted therapies have been proposed, including secondary mutations of the EGFR that prevent/alter either mAb binding or RTK inhibition, activating mutations of the Ras pathway, increased epithelial-mesenchymal transition (EMT), and/or activation of alternative downstream signaling pathways. ${ }^{10,25}$

Elevated concentrations of growth factors and cytokines that activate the STAT3 pathway have been detected in the serum and/or tumor microenvironments of patients with HNSCC. ${ }^{1,5,7,8,26,27}$ For example, transforming growth factor alpha (TGF $\alpha$ )/EGFR autocrine or paracrine signaling in squamous cell carcinoma cells may support the inappropriate or sustained activation of the STAT3 pathway that in turn promotes HNSCC tumor development and/or survival. ${ }^{4,5,8}$ Interleukin-6 (IL-6) is a pleiotropic cytokine that regulates hematopoiesis, inflammation, immune responses, and the production of acute-phase proteins by the liver. IL-6 is produced and secreted by many cancer cells and stromal cell constituents of the tumor microenvironment: tumor infiltrating immune cells ( $\mathrm{T}$ cells, B cells, neutrophils, and macrophages), adipocytes, fibroblasts, and other endothelial or epithelial cell populations. ${ }^{21,22,26,27}$ IL-6 stimulates the growth of many cancer cell types, and elevated serum IL-6 concentrations have been associated with higher cancer stage, faster tumor progression, inhibition of apoptosis, stimulation of angiogenesis, drug resistance, and an adverse prognosis in patients with many types of cancers, including HNSCC, multiple myeloma, lymphoma, ovarian cancer, prostate cancer, lung cancer, breast cancer, metastatic, and renal carcinoma. ${ }^{26,28-35}$ IL-6 was identified as a valuable biomarker for predicting recurrence and overall HNSCC patient survival. ${ }^{26,31,36}$ Tocilizumab, a humanized anti-IL-6R $\alpha$ mAb that blocks IL-6 binding and neutralizes IL-6-induced activities, ${ }^{37,38}$ was approved by the FDA for the treatment of inflammatory diseases such as rheumatoid arthritis, juvenile idiopathic arthritis, Castleman's disease, and Crohn's disease. Tocilizumab and siltuximab, an IL-6 neutralizing antibody, are in clinical trials for multiple myeloma, prostate cancer, and metastatic kidney cancer.

There are currently no small-molecule inhibitors of IL-6 activity in clinical development. We have previously described imaging assays for the development, optimization, and validation of IL-6-induced pSTAT3 and interferongamma (IFN $\gamma$ )-induced pSTAT1 pathway activation in HNSCC cell lines. ${ }^{39}$ These assays were designed to be components of 
a high-content screening (HCS) campaign to identify selective small-molecule inhibitors of STAT3 pathway activation. The current article describes the implementation of these assays to screen a 95,000 compound subset of the NIH Molecular Library Screening Center Network (MLSCN) small-molecule library and the subsequent confirmation and characterization of the selective STAT3 pathway inhibitor hits that were identified.

\section{METHODS AND MATERIALS}

\section{Reagents}

Methanol, formaldehyde, Triton X-100, Tween 20, and Hoechst 33342 were purchased from Sigma-Aldrich (St. Louis, MO). Dimethylsulfoxide (DMSO) (99.9\% high-performance liquid chromatography-grade, under argon) was obtained from Alfa Aesar (Ward Hill, MA). Recombinant human IL-6 and IFN $\gamma$ were purchased from R \& D Systems, Inc., (Minneapolis, MN). The mouse monoclonal anti-pSTAT3 Y705 (Cat\# 612357) and mouse monoclonal anti-pSTAT1-Y701 (Cat\# 612132) primary antibodies were both purchased from Becton Dickenson Biosciences (San Diego, CA). The secondary antibody goat anti mouse-IgG conjugated with Alexa Fluor 488 was purchased from Life Technologies (Grand Island, NY). Dulbecco's $\mathrm{Mg}^{2+}$ and $\mathrm{Ca}^{2+}$-free phosphate-buffered saline (PBS) was purchased from Corning (Tewksbury, MA).

\section{Cells and Tissue Culture}

Four HNSCC cell lines, Cal33, 686LN, FaDu, and OSC19, were utilized in these studies. ${ }^{40-44} \mathrm{Cal} 33$ cells were cultured in Dulbecco's modified Eagle's medium (DMEM) with $2 \mathrm{mM} \mathrm{L-}$ glutamine (Life Technologies) supplemented with 10\% fetal bovine serum (Gemini Bio-Products, West Sacramento, CA), $100 \mu \mathrm{M}$ nonessential amino acids (Life Technologies), $100 \mu \mathrm{M}$ sodium pyruvate (Life Technologies), and $100 \mathrm{U} / \mathrm{mL}$ penicillin and streptomycin (Life Technologies). 686LN cells were cultured in DMEM:F12 supplemented with 10\% FBS, Lglutamine, penicillin, and streptomycin. OSC19 and FaDu cells were cultured in DMEM supplemented with 10\% FBS, nonessential amino acids, L-glutamine, penicillin, and streptomycin.

\section{Compound Libraries}

A 96,491 compound subset of the NIH Molecular Library Screening Center Network (MLSCN) small-molecule collection was solubilized in DMSO at a concentration of $10 \mathrm{mM}$, arrayed into 384-well microtiter master plates, and distributed to the Pittsburgh Molecular Library Screening Center (PMLSC) by the Molecular Library Small-Molecule Repository
(MLSMR), Biofocus-DPI (A Galapagos Company, San Francisco, CA). ${ }^{45-47}$ Compounds were identified by their PubChem substance identity numbers (SIDs). Daughter plates containing $2 \mu \mathrm{L}$ of $10 \mathrm{mM}$ compound solutions in DMSO were replicated from the MLSCN master plates using the Bravo liquid handling platform (Velocity11, Menlo Park, CA) outfitted with a 384-well transfer head. Aluminum adhesive plate seals were applied with an Abgene Seal-IT 100 (Rochester, NY) plate sealer, and plates were stored at $-20^{\circ} \mathrm{C}$ in a Matrical MatriMinistore $^{\mathrm{TM}}$ (Spokane, WA) automated compound storage and retrieval system. For screening, daughter plates were withdrawn from $-20^{\circ} \mathrm{C}$ storage, thawed to ambient temperature, and centrifuged for 1-2 $\mathrm{min}$ at $50 \mathrm{~g}$. The plate seals were removed before the transfer of $98 \mu \mathrm{L}$ of serum-free tissue culture medium (SFM) into wells using the BioTek Microflo liquid handler (BioTek, Winooski, VT) to generate a $200 \mu \mathrm{M}$ intermediate stock concentration of library compound $(2.0 \%$ DMSO). The diluted compounds were mixed by repeated aspiration and dispensation using a 384-well P30 dispensing head on the Evolution-P3 (EP3) liquid handling platform (Perkin Elmer, Waltham, MA), and then $5 \mu \mathrm{L}$ of diluted compounds was transferred to the wells of assay plates. In the primary screen, compounds were individually tested at a final concentration of $20 \mu \mathrm{M}(0.2 \%$ DMSO). After the diluted compounds had been transferred from the daughter plates to the primary HCS assay plates, aluminum adhesive plate seals were applied and the diluted daughter plates were stored frozen at $-20^{\circ} \mathrm{C}$.

Substructure and Similarity Searching of the MLSCN Compound Library to Generate a Cherry-Pick Compound Order for Confirmation

Thirteen HCS priority hits identified in phases I and II (operation runs 1-8) of the HCS campaign were used as template compounds to search the MLSMR compound collection using a Topomer search (3D fragment-based method). An SD file for the 410,748 MLSMR compound collection was downloaded from PubChem and the structures of 96,491 compounds tested in the primary HCS were removed. Compounds that scored below the default Topomer similarity threshold of 185 ( 0 being the most similar) were flagged for further analyses. Each of the lists of structures was reviewed and a manual selection of 20-30 compounds per template was made. Two hundred eighty of the 304 compounds requested from the MLSMR were delivered in the cherry-pick order.

Primary HCS Active Confirmation

Primary HCS actives identified by their PubChem SIDs were requested from the MLSMR and $10 \mu \mathrm{L}$ of $10 \mathrm{mM}$ compounds in 
100\% DMSO was delivered in a separate compound cherrypick order. Daughter plates containing $2 \mu \mathrm{L}$ of $10 \mathrm{mM}$ compounds in DMSO were replicated from the MLSCN cherry-pick master plates using the Bravo outfitted with a 384-well transfer head. Aluminum adhesive plate seals were applied with an Abgene Seal-IT 100 (Rochester, NY) plate sealer and plates were stored at $-20^{\circ} \mathrm{C}$ in a Matrical MatriMinistore ${ }^{\mathrm{TM}}$ automated compound storage and retrieval system. For confirmation, daughter plates were withdrawn from $-20^{\circ} \mathrm{C}$ storage, thawed to ambient temperature, centrifuged for 1-2 min at $50 \mathrm{~g}$, and the plate seals were removed before the transfer of $98 \mu \mathrm{L}$ of SFM into wells using the BioTek Microflo liquid handler to generate a $200 \mu \mathrm{M}$ intermediate stock concentration of library compounds (2.0\% DMSO). The diluted compounds were mixed by repeated aspiration and dispensation using a 384-well P30 dispensing head on the EP3 liquid handling platform, and then $5 \mu \mathrm{L}$ of diluted compounds was transferred to the triplicate wells of assay plates.

For the determination of the 50\% inhibition concentrations ( $\left.\mathrm{IC}_{50}\right), 10$-point 2-fold serial dilutions of test compounds in 100\% DMSO were performed using a 384-well P30 dispensing head on the EP3 liquid handling platform. Daughter plates containing $2 \mu \mathrm{L}$ of the serially diluted compounds in DMSO were prepared and replicated from the 384-well serial dilution master plates using the Bravo outfitted with a 384-well transfer head. Aluminum adhesive plate seals were applied and plates were stored at $-20^{\circ} \mathrm{C}$. For testing in the bioassays, daughter plates were withdrawn from $-20^{\circ} \mathrm{C}$ storage, thawed to ambient temperature, centrifuged for 1$2 \mathrm{~min}$ at $50 \mathrm{~g}$, and the plate seals were removed before the transfer of $38 \mu \mathrm{L}$ of SFM into wells using the BioTek Microflo liquid handler to generate an intermediate stock concentration of library compounds ranging from 1.95 to $500 \mu \mathrm{M}$ (5.0\% DMSO). The diluted compounds were mixed by repeated aspiration and dispensation using a 384-well P30 dispensing head on the EP3, and then $5 \mu \mathrm{L}$ of diluted compounds was transferred to the wells of assay plates to provide a final concentration response ranging from 0.195 to $50 \mu \mathrm{M}$ (0.5\% DMSO).

\section{Automated pSTAT3 and pSTAT1 HCS Assay Protocols}

The automated protocols for the pSTAT3 and pSTAT1 HCS assays have been described previously. ${ }^{39}$ Briefly, Cal33 HNSCC cells were seeded at 2,000 cells per well in $45 \mu \mathrm{L}$ of tissue culture medium into uncoated, black-walled, clear-bottomed (Greiner Bio-one Cat \# 781091) 384-well assay plates on the Zoom dispenser liquid handler (Titertek, Huntsville, AL) and cultured overnight at $37^{\circ} \mathrm{C}, 5 \% \mathrm{CO}_{2}$, and $95 \%$ humidity. Serum- containing medium was then exchanged for SFM on the Bravo liquid handler equipped with a 384-well transfer head. For 24-h compound exposure screens, $5 \mu \mathrm{L}$ of compounds diluted in SFM was transferred to assay plates ( $20 \mu \mathrm{M}, 0.2 \%$ DMSO final) on the Bravo liquid handler equipped with a 384-well transfer head and the treated assay plates were cultured for $24 \mathrm{~h}$ in an incubator at $37^{\circ} \mathrm{C}, 5 \% \mathrm{CO}_{2}$, and $95 \%$ humidity. In the primary screen, $5 \mu \mathrm{L}$ of compounds diluted in SFM was transferred to assay plates ( $20 \mu \mathrm{M}, 0.2 \%$ DMSO final concentration) after the assay plates had been cultured in SFM for $21 \mathrm{~h}$, and the assay plates were placed in an incubator at $37^{\circ} \mathrm{C}, 5 \% \mathrm{CO}_{2}$, and $95 \%$ humidity for an additional $3 \mathrm{~h}$. Compound wells and maximum (Max) control wells were then treated with $50 \mathrm{ng} / \mathrm{mL}$ IL-6 on the Bravo liquid handler equipped with a 384-well transfer head, and minimum (Min) control wells received DMSO (0.2\% final). The assay plate was then centrifuged at $50 \mathrm{~g}$ for $1-2 \mathrm{~min}$, and then returned to the incubator at $37^{\circ} \mathrm{C}, 5 \% \mathrm{CO}_{2}$, and $95 \% \mathrm{hu}-$ midity. After $15 \mathrm{~min}$, the cells were fixed in prewarmed $\left(37^{\circ} \mathrm{C}\right)$ $7.4 \%$ paraformaldehyde containing $2 \mu \mathrm{g} / \mathrm{mL}$ Hoechst 33342 using the BioTek Elx 405 plate washer dispenser. After $30 \mathrm{~min}$ at room temperature, fixed cell monolayers were washed $1 \times$ with PBS, and then the PBS was replaced on the Elx405 plate washer dispenser. The PBS was aspirated, and then fixed cells were permeabilized by the addition of 95\% ice-cold methanol using the Elx405 plate washer dispenser. After $30 \mathrm{~min}$ on ice, the 95\% methanol was removed from the assay plates using the Elx 405 plate washer dispenser, and permeabilized cell monolayers were washed $1 \times$ with Tween 20 blocking buffer, and then the Tween 20 blocking buffer was replaced on the Elx405 plate washer dispenser. Permeabilized cells were incubated in Tween 20 blocking buffer at room temperature for $15 \mathrm{~min}$, and then a 1:100 dilution of mouse anti-pSTAT3-Y705 or mouse antipSTAT1-Y701 primary antibody was added to 384-well assay plates using the Microflo bulk reagent dispenser and incubated for $1 \mathrm{~h}$ in the dark at room temperature. Cell monolayers were then washed $1 \times$ with Tween 20 blocking buffer, and then the Tween 20 blocking buffer was replaced on the Elx405 plate washer dispenser. A 1:1,000 dilution of goat anti-mouse IgG secondary antibody conjugated to Alexa 488 was then added to 384-well assay plates using the Microflo bulk reagent dispenser and incubated for $1 \mathrm{~h}$ in the dark at room temperature. Cell monolayers were washed $1 \times$ with PBS, and then the PBS was replaced on the Elx405 plate washer dispenser, plates were sealed with aluminum foil seals on the Abgene plate sealer, and images were acquired on the ImageXpress Ultra (IXU) automated imaging platform (Molecular Devices, LLC, Sunnyvale, CA). Images were analyzed using the Translocation Enhanced (TE) image analysis module. 
Image Acquisition on the IXU Confocal Automated Imaging Platform

The IXU platform is a fully integrated point-scanning confocal automated imaging platform configured with 4 independent solid-state lasers providing 4 excitation wavelengths of 405, 488, 561, and $635 \mathrm{~nm}$. The IXU was equipped with a Quad filter cube providing emission ranges of 417$477 \mathrm{~nm}, 496-580 \mathrm{~nm}, 553-613 \mathrm{~nm}$, and 645-725 nm and 4 independent photomultiplier tubes (PMTs), each dedicated to a single detection wavelength. The IXU utilizes a dedicated high-speed infrared laser autofocus system, has a 4-position automated objective changer with air objectives $(10 \times, 20 \times$, $40 \times$, and $60 \times$ ), and the detection pinhole diameter of the confocal optics was configurable in the software. For the pSTAT3 and pSTAT1 HCS assays, the IXU was set up to acquire 2 images using a $20 \times 0.45$ NA ELWD objective in each of 2 fluorescent channels, which were acquired sequentially. ${ }^{39}$ For the studies describe herein, the Hoechst channel laser autofocus Z-offset was $-6.98 \mu \mathrm{m}$, the 405 laser was set at 10\% power, and the PMT gain was 550. The pSTAT3-Y705 FITC channel Z-offset from W1 (the Hoechst channel) was $12.96 \mu \mathrm{m}$, the 488 laser was set at 10\% power, and the PMT gain was 625. On average, the IXU scanned a single 384-well plate, 2 images per channel, in 90 min using these settings.

\section{Image Analysis Using the TE Module}

Hoechst 33342 was used to stain and identify the nucleus, and this fluorescent signal from channel 1 (Ch 1) was used by the TE module to define an inner nuclear mask and an outer cytoplasm mask in channel 2 (Ch 2). ${ }^{39}$ Objects in $\mathrm{Ch} 1$ that exhibited the appropriate fluorescent intensities above background and size (width, length, and area) characteristics were identified and classified by the image segmentation as nuclei. For Hoechst-stained Cal33 cells, the following settings typically proved effective: objects defined as nuclei had to exhibit fluorescent intensities $>1500$ gray levels over background, and they had to have an approximate width of $10 \mu \mathrm{m}$ with a minimum area of $20 \mu^{2}$, and not to exceed a maximum area of $1000 \mu \mathrm{m}^{2}$. Objects that met these criteria were used to create nuclear masks for each cell. The nuclear mask was eroded by $1 \mu \mathrm{m}$ in from the edge of the detected nucleus to reduce cytoplasmic contamination within the nuclear area, and the reduced inner mask was used to quantify the amount of target channel (Ch 2) fluorescence (pSTAT3-Y705 or pSTAT1-Y701) within the nucleus. The outer cytoplasm mask was then established $1 \mu \mathrm{m}$ out from the edge of the detected nucleus, and the width of the outer mask was set at $3 \mu \mathrm{m}$ to cover as much of the cytoplasm region as possible without going outside the cell boundary. The outer mask was used to quantify the amount of target Ch 2 fluorescence (pSTAT3-Y705 or pSTAT1-Y701) within the cytoplasm. The TE module outputs quantitative data, such as the average fluorescent intensities of the Hoechst-stained objects in $\mathrm{Ch} \mathrm{1,} \mathrm{the} \mathrm{selected} \mathrm{object} \mathrm{or}$ cell count in $\mathrm{Ch} 1$, the integrated and average fluorescent intensities of the Ch2 signal in the nucleus (inner) or cytoplasm (outer) regions as an overall well average value, or on an individual cell basis. The average fluorescent intensities of the pSTAT3 or pSTAT1 Ch2 signals quantified by the TE module were used to measure activation of the STAT3 and STAT1 signaling pathways, respectively. ${ }^{39}$

\section{Cytotoxic Compound and Fluorescent Outlier Analysis}

To identify and eliminate cytotoxic and fluorescent compounds, we calculated $\mathrm{z}$-scores for several multiparameter data outputs from the TE module as described previous$1 y^{39,45,48-53}: z=(X-\mu) / \sigma$, where $z$ is the $z$-score, $X$ is the parameter value of a single compound-treated well, $\mu$ is the population mean parameter value of all 320 compoundtreated wells on the assay plate, and $\sigma$ is the standard deviation of the mean parameter value. For cytotoxic compounds, we used the average number of Hoechst-stained nuclei per image parameter, and used a threshold z-score of $\leq-4$ to flag compounds that were either cytotoxic or reduced Cal33 cell adherence sufficiently that they should be excluded. To identify and eliminate compounds that fluoresced in Ch1, we calculated z-scores for the mean nuclear average intensities of the Hoechst-stained nuclei parameter and set a threshold zscore of $\geq 4$ to flag compounds that fluoresced in $\mathrm{Ch} 1$ and should be excluded. To identify and eliminate compounds that fluoresced in Ch2, we calculated z-scores for both the integrated and average outer (cytoplasm) intensity of the Ch2 FITC signal in the cytoplasm region of cells and set threshold z-scores of $\geq 4$ to flag compounds that fluoresced within cells in Ch2 and should be excluded.

\section{Head and Neck Squamous Cell Carcinoma Cell Line} Growth Inhibition Assays

The HNSCC cell line growth inhibition assays have been described previously. ${ }^{39}$ On day 1 , each cell line was harvested, counted, and seeded into two 384-well assay plates, a time zero (T0) and a time $72 \mathrm{~h}$ (T72) plate. Cal33, 686LN, FaDu, and OSC19 HNSCC cells were seeded at 1,000 cells per well in $45 \mu \mathrm{L}$ of tissue culture media into uncoated, white, opaque 384-well assay plates (Greiner Bio-one Cat \# 781080/1B) on the Zoom dispenser liquid handler or the BioTek Microflo liquid handler and cultured overnight at $37^{\circ} \mathrm{C}, 5 \% \mathrm{CO}_{2}$, and 95\% humidity. On day 2, $25 \mu \mathrm{L}$ of the CellTiter-Glo (CTG) (Promega Corporation, Madison, WI) detection reagent was 
dispensed into the wells of the To assay plate on the BioTek Microflo liquid handler, and the luminescence signal was captured on the Envision (Perkin Elmer, Waltham, MA) microtiter plate reader. In addition, on day 2, $5 \mu \mathrm{L}$ of compounds diluted in SFM was transferred into the test wells of the T72 384-well assay plates on the Bravo liquid handler equipped with a 384-well transfer head, and the compound-treated assay plates were cultured for $72 \mathrm{~h}$ in an incubator at $37^{\circ} \mathrm{C}, 5 \%$ $\mathrm{CO}_{2}$, and 95\% humidity. Control wells received DMSO alone. On day 5, $25 \mu \mathrm{L}$ of the CTG detection reagent was dispensed into the wells of the T72 assay plate on the BioTek Microflo liquid handler, and the luminescence signal was captured on the Envision microtiter plate reader platform.

Data Processing, Visualization, Statistical Analysis, and Curve Fitting

HCS data processing for the pSTAT activation screens and the HNSCC growth inhibition assays were performed using ActivityBase ${ }^{\mathrm{TM}}$ (IDBS, Guildford, UK) and CytoMiner (UPDDI). Processed data and HCS multiparameter features were visualized using Spotfire ${ }^{\mathrm{TM}}$ DecisionSite $^{\mathrm{TM}}$ (Somerville, MA) software. ${ }^{45,48,49}$ An ActivityBase primary HTS template was created that automatically calculated \% inhibition together with plate control signal-to-background (S:B) ratios and Z'-factor coefficients. We utilized the mean average inner intensity values (pSTAT3-Y705 or pSTAT1-Y701) of the $0.2 \%$ DMSO minimum plate control wells $(n=32)$ and the mean average inner intensity values of the $50 \mathrm{ng} / \mathrm{mL}$ IL-6 (pSTAT3-Y705) or $30 \mathrm{ng} / \mathrm{mL}$ IFN $\gamma$ (pSTAT1-Y701) maximum plate control wells $(n=32)$ to normalize the mean average inner intensity values (pSTAT3-Y705 or pSTAT1-Y701) of the compound data and to represent $0 \%$ and 100\% activation/translocation of pSTAT3 or pSTAT1 to the nucleus, respectively.

For the HNSCC growth inhibition assays, we used the DMSO control data from the T0 and T72 assay plates to assess the dynamic range of the T0 to T72 cell growth, and to calculate $\mathrm{S}: \mathrm{B}$ ratios and Z'-factor coefficient statistics for the assay signal window (T0 to T72). To normalize the 72-h compound exposure HNSCC growth inhibition data, the signals from the compound-treated wells were processed and expressed as \% of the T72 DMSO plate controls.

We also constructed an ActivityBase concentrationresponse template to calculate percent inhibition together with plate control S:B ratios and Z'-factor coefficients for quality control purposes. ${ }^{39,45-49} \mathrm{IC}_{50}$ values were calculated using an XLfit 4-parameter logistic model, also called the Sigmoidal dose-response model: $y=(A+((B-A) /(1+((C / x) D))))$, where $\mathrm{y}$ was the percent activation and $\mathrm{x}$ was the corresponding compound concentration. The fitted C parameter was the $\mathrm{IC}_{50}$ and defined as the concentration giving a response halfway between the fitted top (B) and bottom (A) of the curve, and D is the slope factor. For normalized data, the A and B parameters were locked as 0 and 100, respectively. For non-normalized concentration response data, we used GraphPad Prism 5 software to plot and fit data to curves using the Sigmoidal dose-response variable slope equation: $\mathrm{Y}=$ Bottom $+[$ Top - Bottom $] /\left[1+10^{\wedge}\left(\operatorname{LogEC}_{50}-\mathrm{X}\right)^{*}\right.$ HillSlope $]$.

\section{RESULTS}

\section{Primary HCS Campaign}

We utilized a 384-well pSTAT3 pathway activation HCS assay that we previously developed and validated ${ }^{39}$ to screen a 94,491 compound subset of the NIH MLSCN library for small molecules that inhibited IL-6-induced pSTAT3-Y705 activation in the Cal33 HNSCC cell line (Figs. 1-3 and Table 1). Cal33 cells that had been serum starved overnight were preexposed to $20 \mu \mathrm{M}$ compounds for $3 \mathrm{~h}$ before activation of the STAT3 signaling pathway by treatment with $50 \mathrm{ng} / \mathrm{mL}$ IL-6 for $15 \mathrm{~min}$. Each $384-$ well assay plate contained $32 \times$ maximum (Max, IL-6+DMSO) and $32 \times$ minimum (Min, DMSO) plate controls that were used to normalize the pSTAT3-Y705 responses of the 320 test compound wells and to derive signalto-background (S:B) ratio and Z'-factor coefficient assay performance quality control statistics for the HCS campaign (Figs. 1-3). Figure 1A shows representative 20X grayscale and color composite images of Hoechst-stained Cal33 nuclei (Ch1) and anti-pSTAT3-Y705 indirect immunofluorescent FITC staining (Ch2) of IL-6 Max and DMSO Min plate controls acquired on the IXU automated confocal imaging platform. IL-6 treatment dramatically increased the pSTAT3-Y705 fluorescent signal predominantly localized within the nuclei of Cal33 cells as indicated by the colocalization of the Hoechst and FITC signals in the color composite images of IL-6-treated cells (Fig. 1A). We used the TE module of the IXU platform to extract multiparameter quantitative data from these digital images, as previously described, and Figure $1 B$ and $C$ represents the average fluorescent intensities of the Ch2 pSTAT3Y705 signal in the nuclei of Cal33 cells and the Ch1 nuclei count ( = average number of cells per image), respectively, for the Max and Min plate controls of a typical HCS assay plate. Consistent with the images presented in Figure 1A, IL-6 treatment induced a significant increase in the average inner (nuclear) intensity of the pSTAT3-Y705 fluorescent signal over DMSO controls without significantly altering the number of cells per image (Fig. 1B, C). The average inner (nuclear) intensity of the pSTAT3-Y705 fluorescent signal from the $32 \times$ Max and $32 \times$ Min plate controls provided a robust and 


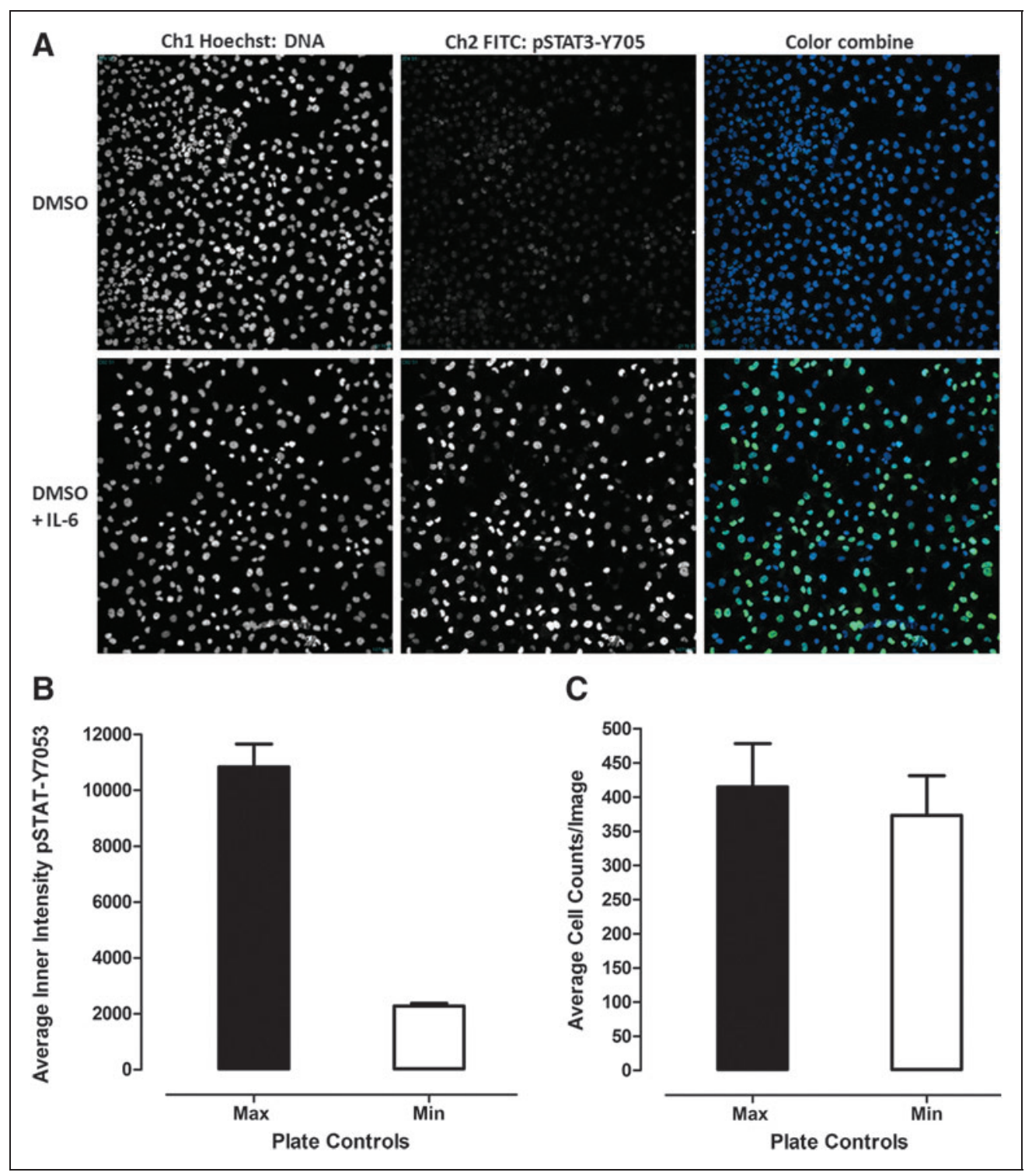

Fig. 1. Interleukin-6 (IL-6)-induced pSTAT3 activation high-content screening (HCS) assay. (A) Representative grayscale and color composite images of HCS plate controls, Cal33 cells \pm IL-6 treatment. Grayscale images of Hoechst 33342-stained nuclei (Ch 1) and pSTAT3-Y705 staining (Ch 2), and color composite images of control (0.2\% DMSO) and IL-6+DMSO-treated ( $50 \mathrm{ng} / \mathrm{mL}, 15 \mathrm{~min}$ ) Cal 33 head and neck squamous cell carcinoma (HNSCC) cells. (B, C) Quantitative data extracted from the digital images of the Hoechst-stained nuclei and pSTAT3-Y705 Staining in HCS plate controls, Cal33 cells \pm IL- 6 treatment, by the translocation enhanced (TE) module; (B) Average pSTAT3-Y705 intensity in the inner nuclear mask area; (C) Cell counts per image. Three hundred eighty-four well plates were seeded with Cal33 HNSCC cells and serum starved for $24 \mathrm{~h}$ as described in the Materials and Methods section. After $24 \mathrm{~h}$, Cal33 cells were treated with $0.2 \% \mathrm{DMSO}$ ( $\square$ ) or $50 \mathrm{ng} / \mathrm{mL}$ of IL$6+0.2 \%$ DMSO ( $\square$ ) for $15 \mathrm{~min}$ at $37^{\circ} \mathrm{C}$ in $5 \% \mathrm{CO}_{2}$ and $95 \%$ humidity and were then fixed and stained with Hoechst and a mouse monoclonal anti-p-STAT3-Y705 antibody as described in the Materials and Methods section. Images were acquired on the ImageXpress Ultra (IXU) confocal HCS platform and analyzed using the TE module as described in the Materials and Methods section. The grayscale and color composite images presented are representative of similar images obtained in numerous independent experiments. The quantitative data represent the mean \pm SD of 32 Max (IL-6+DMSO) and 32 Min (DMSO) plate control wells from one of these independent experiments.

reproducible assay signal window for IL-6-induced STAT3 pathway activation as indicated by the corresponding $\mathrm{S}: \mathrm{B}$ ratio of 4.76-fold and $\mathrm{Z}^{\prime}$-factor coefficient of 0.68 (Fig. 1B).

Figure $2 \mathrm{~A}$ is a scatterplot of the pSTAT3-Y705 average inner (nuclear) intensity raw data of a single representative 384-well assay plate from the primary HCS campaign. The pSTAT3-Y705 average inner intensity responses of the IL-6treated Max controls were $\sim 5$-fold higher and well separated from the DMSO controls, and most of the compound-treated wells produced signals similar to the Max controls, indicating 
that they failed to inhibit IL-6-induced STAT3 pathway activation (Fig. 2A). Two of the 320 compounds on this assay plate did, however, significantly inhibit the IL-6-induced pSTAT3-Y705 average inner (nuclear) intensity signal (Fig. $2 A$ ). Figure $2 B$ is an overlay scatterplot of the normalized $\%$

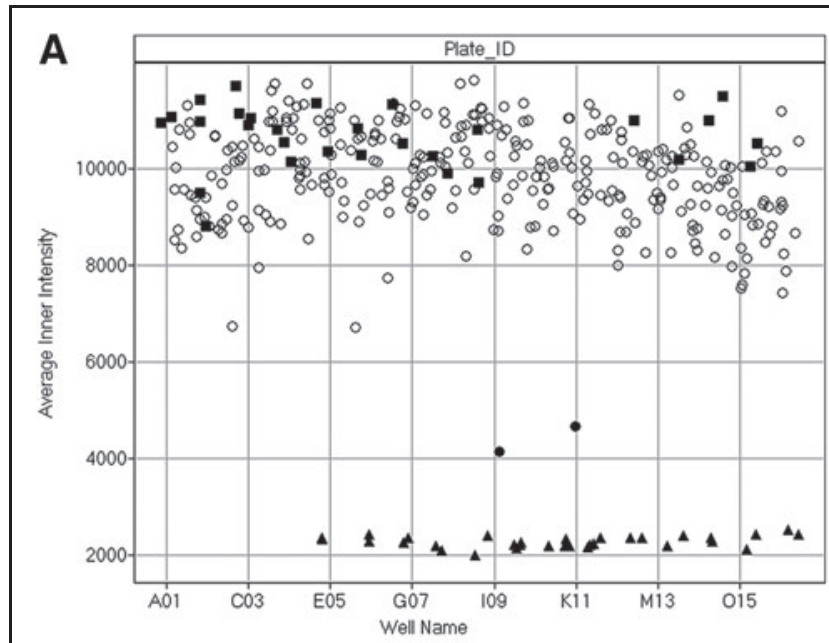

pSTAT3 HCS Operations Run: $19 \times 384$-well Plate Overlay of $\%$ Inhibition Scatterplot

B

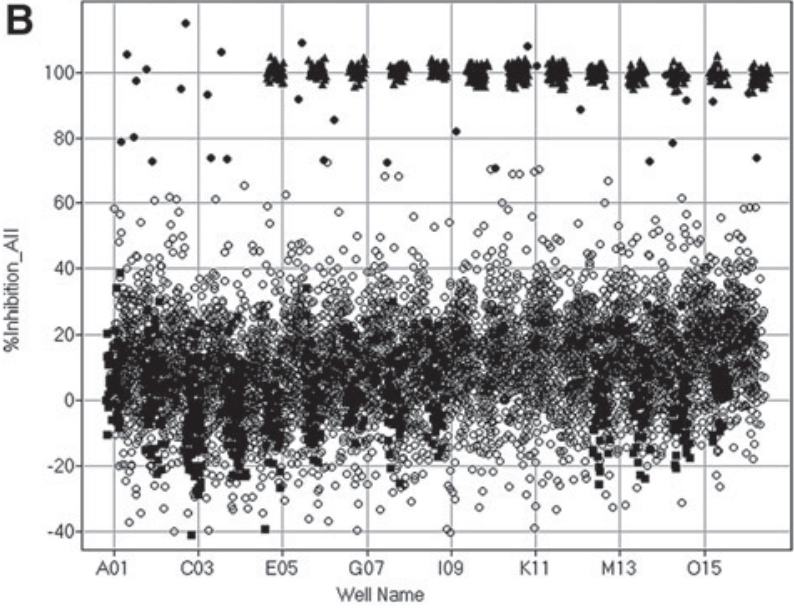

C $\%$ Inhibition Average Inner Intensity PSTAT3-Y705

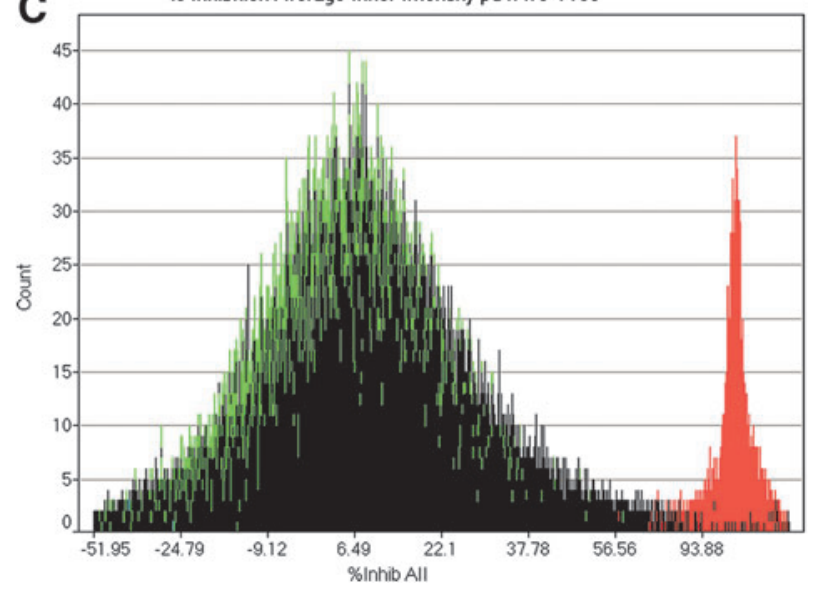

inhibition data from a single screening operation run of 6,080 compounds that were arrayed and screened at $20 \mu \mathrm{M}$ in $19 \times 384$-well assay plates. The normalized pSTAT3-Y705 average inner intensity responses of the Max and Min plate controls were reproducible and provided a robust assay signal window (Fig. 2B). Although the majority of the compounds tested failed to inhibit the IL-6-induced activation of the STAT3 pathway, 30 compounds achieved the activity criterion of $\geq 70 \%$ inhibition of IL-6-induced pSTAT3 activation (Fig. 2B). A frequency distribution plot of the normalized \%

Fig. 2. Primary HCS to identify inhibitors of IL-6-induced pSTAT3 activation. (A) Scatterplot of the pSTAT3-Y705 intensity data from a single representative HCS assay plate. The average inner PSTAT3Y705 intensity data from a single 384 -well assay plate from the HCS campaign are presented; IL-6 Max (ם) controls, DMSO Min (A) controls, inactive $(<70 \%$ inhibition) compound-treated wells (O), and active ( $\geq 70 \%$ inhibition) compound-treated wells (O). (B) Overlay scatterplot of the normalized \% inhibition data from $19 \times 384$-well assay plates from a representative screening operation run. The mean average inner pSTAT3-Y705 intensity values of the $0.2 \%$ DMSO Min plate control wells $(n=32)$ and the $50 \mathrm{ng} / \mathrm{mL}$ IL-6 Max plate control wells $(n=32)$ were used to normalize the mean average inner intensity pSTAT3-Y705 values of the compound-treated wells and to represent $100 \%$ and $0 \%$ inhibition of IL-6-induced pSTAT3 activation, respectively. An overlay scatterplot of the normalized \% inhibition data from a single operation run of $19 \times 384$-well assay plates from the HCS campaign is presented; IL-6 Max $(\boldsymbol{\square})$ controls, DMSO Min $(\mathbf{\Delta})$ controls, inactive ( $<70 \%$ inhibition) compound-treated wells $(O)$, and active $(\geq 70 \%$ inhibition) compound-treated wells (O). (C) Binned results frequency distribution graph of the normalized \% inhibition data from the primary HCS. The mean average inner pSTAT3-Y705 intensity values of the $0.2 \%$ DMSO Min plate control wells $(n=32)$ and the $50 \mathrm{ng} / \mathrm{mL}$ IL-6 Max plate control wells $(n=32)$ were used to normalize the mean average inner intensity pSTAT3-Y705 values of the compound-treated wells and to represent $100 \%$ and $0 \%$ inhibition of IL-6-induced pSTAT3 activation, respectively. The normalized \% inhibition data from the $294 \times 384$-well assay plates from the primary HCS were binned into discrete ranges and the counts within each bin are presented; IL-6 Max (green) controls, DMSO Min (red) controls, inactive $(<70 \%$ inhibition) compound-treated wells (black), and active ( $\geq 70 \%$ inhibition) compound-treated wells (light blue). Three hundred eighty-four well plates were seeded with Cal33 HNSCC cells and serum starved for $24 \mathrm{~h}$ as described in the Materials and Methods section. After $24 \mathrm{~h}$, compounds were transferred from compound library daughter plates diluted in SFM into the assay plates to provide a final screening concentration of $20 \mu \mathrm{M}$ (0.2\% DMSO), and then incubated at $37^{\circ} \mathrm{C}, 5 \% \mathrm{CO}_{2}$, and $95 \%$ humidity for $3 \mathrm{~h}$. Compound-treated wells and Max plate controls then received $50 \mathrm{ng} / \mathrm{mL} \mathrm{IL}-6$ in $0.2 \%$ DMSO (final) and Min controls received $0.2 \%$ DMSO (final) in the medium, and assay plates were then incubated at $37^{\circ} \mathrm{C}, 5 \% \mathrm{CO}_{2}$, and $95 \%$ humidity for $15 \mathrm{~min}$ and then fixed and stained with Hoechst and a mouse monoclonal anti-p-STAT3-Y705 antibody as described in the Materials and Methods section. Images were acquired on the IXU and analyzed using the TE module as described in the Materials and Methods section. 


\section{JOHNSTON ET AL.}

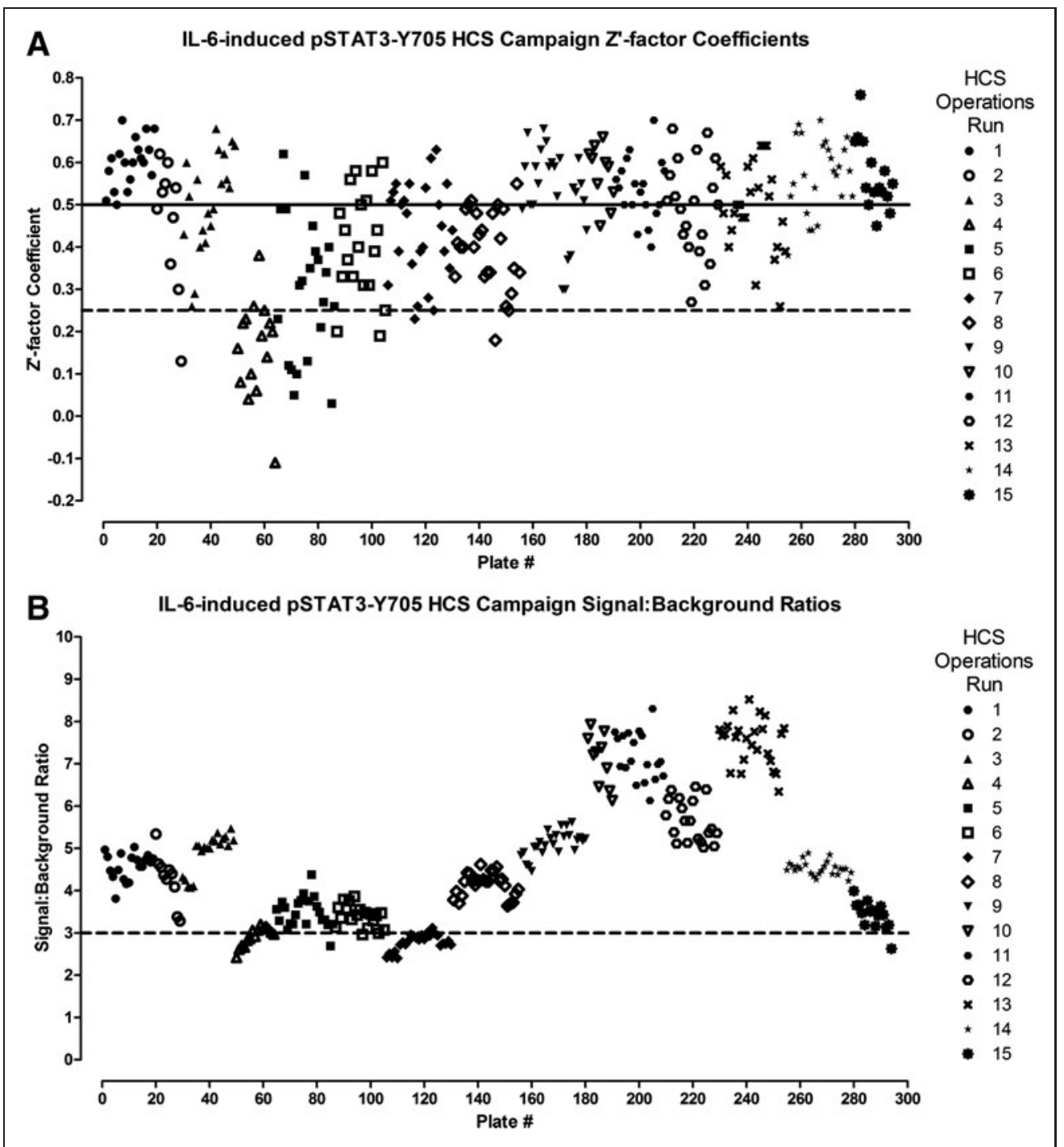

Fig. 3. IL-6-induced pSTAT3 pathway activation HCS campaign performance statistics (A) Plate Z'-factor coefficients and (B) signal:background ratios. The mean average inner pSTAT3-Y705 intensity values of the $0.2 \%$ DMSO Min plate control wells $(n=32)$ and the $50 \mathrm{ng} / \mathrm{mL}$ IL-6 Max plate control wells $(n=32)$ from each of the $294 \times 384$-well assay plates in the primary HCS were used to calculate Z'-factor coefficients and signal:background (S:B) ratios for quality control review purposes. Eleven assay plates that exhibited a Z'-factor coefficient $<0.25$ and an S:B ratio $<3$ were failed and scheduled for retesting.

inhibition data for the controls and compound-treated wells for the 94,491 compound primary HCS campaign illustrates that the population responses of the Max and Min controls were distinct and that $98.6 \%$ of the compounds exhibited IL6-induced pSTAT3-Y705 average inner intensity responses comparable with Max controls and were therefore flagged as inactive (Fig. 2C). Only 1,307 (1.4\%) of the compounds screened at $20 \mu \mathrm{M}$ achieved the activity criterion of $\geq 70 \%$ inhibition (Fig. 2C and Table 1).

To further illustrate the performance of the IL-6-induced pSTAT3 activation assay during the primary HCS campaign, we present the $Z^{\prime}$-factor coefficient and S:B ratio assay performance statistics for all 294 assay plates screened on 15 separate operation days spanning a period of several months and conducted in 3 distinct phases (Fig. 3A, B). Before implementing the screen, we had established the following quality control metrics for the performance of the plate controls ${ }^{39}$ : plates that exhibited a $Z^{\prime}$-factor coefficient $<0.25$ and an $\mathrm{S}: \mathrm{B}$ ratio $<3$ would be failed and retested. The pSTAT3 pathway activation assay performed well during screening and, on average, exhibited $Z^{\prime}$-factor coefficients of $0.47 \pm 0.15$ $(n=294)$ (Fig. 3A) with average S:B ratios of $4.71 \pm 1.52$ 
Table 1. Inhibition of IL-6-Induced pSTAT3 Activation Primary HCS Summary

pSTAT3 HCS multiparameter Number of data and criteria compounds total

Total number of compounds

$\geq 70 \%$ inhibition of IL-6-induced pSTAT3 activation

Average cell counts per image $z$-score $\leq-4^{\text {a }}$

Ch1 mean nuclear average intensity $z$-score $\geq 4^{b}$

Ch1 mean nuclear average intensity $z$-score $\leq-4^{b}$

Ch2 integrated outer intensity $z$-score $\geq 4^{c}$

Ch2 integrated outer intensity $z$-score $\leq-4^{c}$

Ch2 average outer intensity z-score $\geq 4^{d}$

Ch2 average outer intensity z-score $\leq-4^{d}$

Primary HCS actives

94,491

1,307

207

252

17

271

Cal33 HNC cells that had been cultured overnight under serum-free conditions in an incubator at $37^{\circ} \mathrm{C}, 5 \% \mathrm{CO}_{2}$, and $95 \%$ humidity were preexposed to the diluted compound library at a final concentration of $20 \mu \mathrm{M}$ ( $0.2 \%$ DMSO) for $3 \mathrm{~h}$ before activation of the pSTAT3 signaling pathway by the addition of $50 \mathrm{ng} / \mathrm{mL} \mathrm{IL-6}$ for $15 \mathrm{~min}$. Cells were then fixed and stained for nuclear DNA and PSTAT3-Y705 levels, 20X images were acquired on the IXU confocal automated HCS platform, and the average nuclear intensity of pSTAT3Y705 was quantified using the TE image analysis module. The average nuclear intensity of pSTAT3-Y705 from compound-treated wells was normalized relative to 32 maximum (IL-6) and minimum (DMSO) plate controls on the same plate and the \% inhibition of IL-6-induced pSTAT3 activation was calculated. In addition, several of the multiparameter data outputs from the TE image analysis module were used to identify and eliminate cytotoxic compounds or compounds that were fluorescent outliers.

${ }^{a}$ Compounds that exhibited average cell counts per image $z$-scores $\leq-4$ were flagged as cytotoxic outliers.

${ }^{b}$ Compounds that exhibited mean average intensity z-scores $\geq 4$ or $\leq-4$ in the Hoechst channel were flagged as fluorescent outliers in Ch1.

'Compounds that exhibited mean integrated intensity $z$-scores $\geq 4$ or $\leq-4$ in the outer (cytoplasm) region of the Alexa 488 channel were flagged as fluorescent outliers in Ch2.

${ }^{d}$ Compounds that exhibited mean average intensity $z$-scores $\geq 4$ or $\leq-4$ in the outer (cytoplasm) region of the Alexa 488 channel were flagged as fluorescent outliers in Ch2.

$(n=294)$ (Fig. 3B). However, eleven assay plates from the fourth and fifth screening operation runs produced Z'-factor coefficients $<0.25$ with S:B ratios $<3$ and were retested. To troubleshoot the pSTAT3 activation assay, we conducted a series of experiments to evaluate and compare the relative contributions of critical assay components to the dynamic range and reproducibility of the assay: the passage \# of the Cal33 cells, the anti-pSTAT3-Y705 antibody lot, and the length of time in storage at $-80^{\circ} \mathrm{C}$ of the reconstituted IL- 6 (Fig. 4). There did not appear to be any significant differences between data generated with the lot of the anti-pSTAT3-Y705 antibody used for the assay development studies and the large single lot purchased for the HCS campaign (Fig. 4A, B vs. C, $D$ ). In comparison with a lot of IL-6 that had been reconstituted and stored at $-80^{\circ} \mathrm{C}$ for several weeks, a newly reconstituted lot of IL-6 produced larger pSTAT3-Y705 average inner intensity signals at lower ( 30 and $40 \mathrm{ng} / \mathrm{mL}$ ) cytokine concentrations (Fig. $4 A, B$ vs. C, D). However, it was the passage number of the Cal33 cell line that was shown to be the major factor that impacted the performance of the assay (Fig. 4). Cal33 cells from passage 8 exhibited a more robust IL-6induced pSTAT3 activation response than passage 24 cells, independently of the antibody and the IL- 6 reagents utilized (Fig. 4). Based on these data, the remaining HCS operation runs (6-15) were conducted in Cal33 cells with passage \#s $<20$ and with IL-6 that had been reconstituted and stored at $-80^{\circ} \mathrm{C}$ for $\leq 4$ weeks.

To identify and eliminate cytotoxic and autofluorescent compounds that might be flagged as false positives or could interfere with the pSTAT3 activation assay format, we calculated z-scores for several of the parameters output by the TE module (Table 1 and Supplementary Figs. S1 and S2; Supplementary Data are available online at www.liebertpub.com/ adt), as described previously ${ }^{39,45,48-53} ; 207(0.22 \%)$ of the compounds screened were either cytotoxic or reduced Cal33 cell adherence sufficiently that after $3 \mathrm{~h}$ at $20 \mu \mathrm{M}$ they exhibited average cell counts per image z-scores $\leq-4$ (Table 1 and Supplementary Fig. S1A). Representative Ch1 images of Hoechst-stained nuclei from these wells illustrate the apparent loss of cells compared with plate controls (Supplementary Fig. $S 2 A)$. Two hundred fifty-two compounds $(0.27 \%)$ significantly increased the mean average Hoechst-stained nuclear intensity values in Ch1 and produced z-scores $>4$ (Table 1 and Supplementary Fig. S1B). Representative Ch1 images show that these compounds fluoresce strongly when bound to DNA (Supplementary Fig. S2B) and will therefore interfere with the ability of the image analysis algorithm to accurately create the nuclear region of interest in which to quantify pSTAT3 activation in Ch2 images. In general, compounds with mean average Hoechst-stained nuclear intensity $z$-scores $<-4$ were also flagged for cytotoxicity. Two hundred and seventy-one $(0.29 \%)$ and $453(0.48 \%)$ of the compounds screened produced z-scores $>4$ for the Ch2 FITC integrated and average outer (cytoplasm) intensity values, respectively (Table 1 and Supplementary Fig. S1C and D). Representative Ch2 images indicate that these compounds fluoresce brightly in the FITC channel within cells (Supplementary Fig. S2C) and will 


\section{JOHNSTON ET AL.}

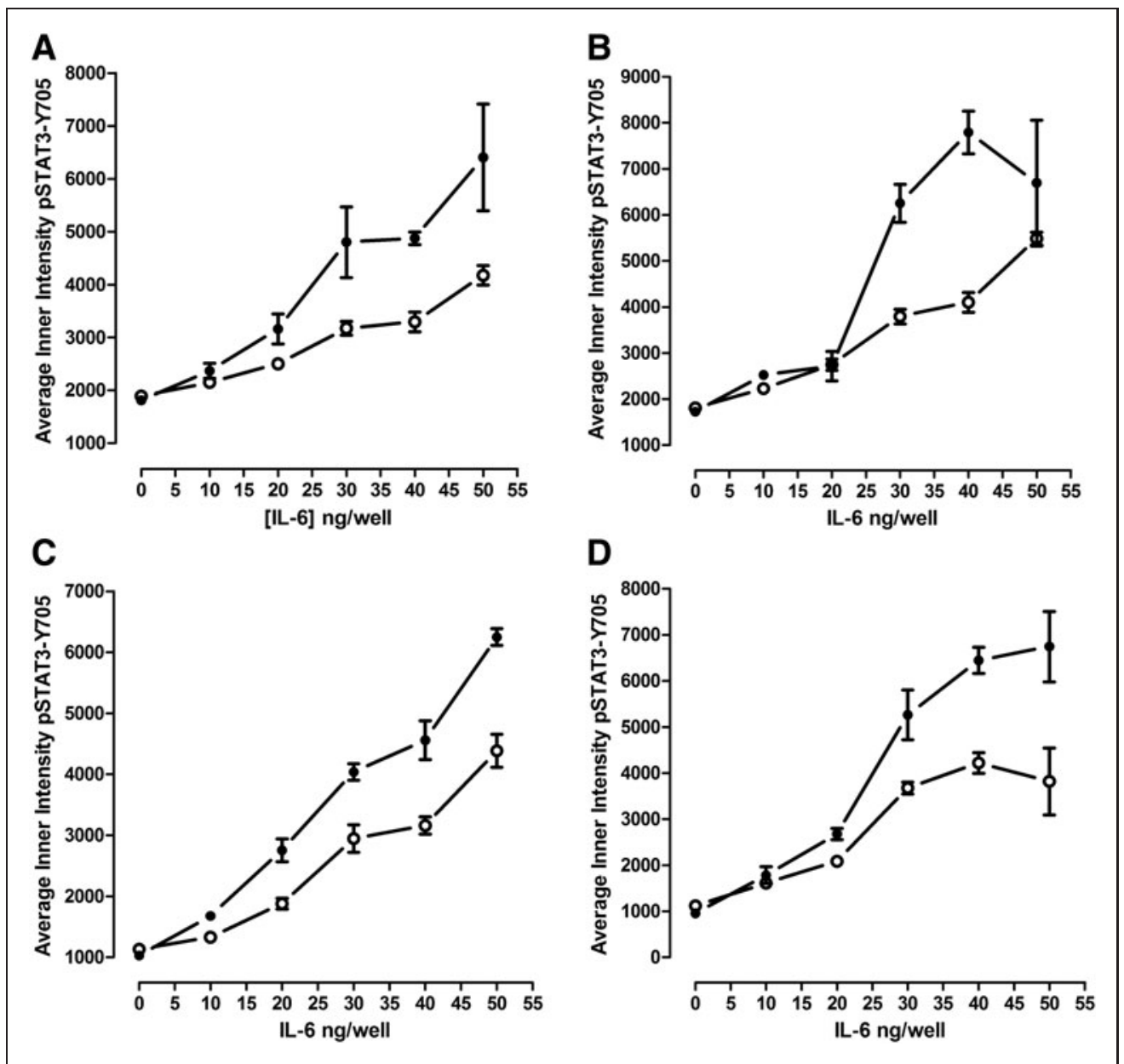

Fig. 4. Troubleshooting the IL-6-induced pSTAT3 activation HCS assay. (A) Comparison of the IL-6-induced pSTAT3-Y705 signals in Cal33 cells of a different passage number using the anti-p-STAT3-Y705 antibody lot procured for the HCS campaign and IL-6 that had been reconstituted and stored in aliquots at $-80^{\circ} \mathrm{C}$. (B) Comparison of the IL-6-induced pSTAT3-Y705 signals in Cal 33 cells of different passage number using the anti-p-STAT3-Y705 antibody lot procured for the HCS campaign and freshly reconstituted IL-6. (C) Comparison of the IL-6induced pSTAT3-Y705 signals in Cal33 cells of different passage number using the anti-p-STAT3-Y705 antibody lot previously procured for assay development and IL- 6 that had been reconstituted and stored in aliquots at $-80^{\circ} \mathrm{C}$. (D) Comparison of the IL-6-induced pSTAT3-Y705 signals in $\mathrm{Cal}_{33}$ cells of different passage number using the anti-p-STAT3-Y705 antibody lot previously procured for assay development and freshly reconstituted IL-6. Cal33 HNSCC cells were seeded into assay plates and serum starved for $24 \mathrm{~h}$ as described in the Materials and Methods section. After $24 \mathrm{~h}$, Cal33 cells were treated with $0.2 \%$ DMSO or the indicated concentrations of IL-6 $+0.2 \%$ DMSO for 15 min at $37^{\circ} \mathrm{C}$ in $5 \% \mathrm{CO}_{2}$ and $95 \%$ humidity and were then fixed and stained with Hoechst and a mouse monoclonal anti-p-STAT3-Y705 antibody as described in the Materials and Methods section. Images were acquired on the IXU and analyzed using the TE module as described in the Materials and Methods section. The mean \pm SD $(n=3)$ average inner (nuclear) intensities of the p-STAT3-Y70 5 signals from 3 wells of Cal33 cells, passage \# $8(\bigcirc)$ or passage \# $24(\bigcirc)$, treated with each cytokine concentration are presented. Representative experimental data from one of 2 independent experiments are shown.

therefore obscure any compound effects on IL-6-induced pSTAT3-Y705 activation. Fewer compounds produced zscores $<-4$ for the Ch2 FITC integrated (122) and average (34) outer intensity values (Table 1) and the majority of these were also flagged for cell loss. From the 1,307 compounds that inhibited IL-6-induced pSTAT3 activation by $\geq 70 \%$ at $20 \mu \mathrm{M}$ (Fig. 2C), a total of 1,068 actives remained after we had eliminated cytotoxic and autofluorescent compounds that interfered with the pSTAT3 HCS assay format (Table 1).

\section{Primary HCS Active Confirmation}

The STAT3 pathway inhibitor primary HCS campaign was prosecuted in 3 distinct phases; the first screening operation run was completed in phase I, screening operation runs 2-8 
were completed in phase II, and runs 9-15 were completed in phase III. Seven hundred twenty-eight active compounds identified in phases I and II that were not either acutely cytotoxic or fluorescent outliers were ordered from the MLSMR (Table 2). Only 668 of the requested compounds were available for cherry-picking and 32 of these samples failed the
SMR quality control criterion because they were $<90 \%$ pure by LC/MS analysis (Table 2); 222 (33.2\%) of the 668 samples were confirmed active because they inhibited IL-6-induced pSTAT3 activation $\geq 70 \%$ at $20 \mu \mathrm{M}(n=3)$, and 126 also inhibited IFN $\gamma$-induced pSTAT1 activation by $\geq 50 \%$ at $20 \mu \mathrm{M}$ $(n=3)$ (Table 2). All of the confirmed pSTAT3 pathway

\begin{tabular}{|c|c|c|c|c|}
\hline \multirow[t]{2}{*}{ pSTAT3 Primary HCS confirmation } & \multicolumn{2}{|c|}{ Phases I and II } & \multicolumn{2}{|l|}{ Phase III } \\
\hline & \multicolumn{2}{|c|}{ Operation runs $1-8$} & \multicolumn{2}{|c|}{ Operation runs $9-15$} \\
\hline pSTAT3 confirmation & Number of compounds & $\%$ of total ${ }^{a}$ & Number of compounds & $\%$ of total ${ }^{a}$ \\
\hline Total number of primary HCS actives identified ${ }^{b}$ & 728 & 100 & 279 & \\
\hline Total number of compounds requested from the SMR ${ }^{d}$ & 728 & 100 & 583 & $100(583)$ \\
\hline Number of cherry-pick samples supplied by SMR ${ }^{e}$ & 668 & $91.8(728)$ & 519 & $89(583)$ \\
\hline Number of samples failed SMR OC $<90 \%$ pure $^{f}$ & 32 & $4.8(668)$ & 9 & $1.7(519)$ \\
\hline Mean $\%$ inhibition of IL-6-induced pSTAT3 $\geq 70 \%(n=3)^{g}$ & 222 & $33.2(668)$ & & \\
\hline Inhibition of IL-6-induced pSTAT3 $\mathrm{IC}_{50}<50 \mu \mathrm{M}^{\mathrm{i}}$ & 136 & $61.3(222)$ & 67 & $38.5(174)$ \\
\hline Inhibition of IFN $\gamma$-induced pSTAT1 $\mathrm{IC}_{50}<50 \mu \mathrm{M}^{\mathrm{i}}$ & 53 & $23.9(222)$ & 36 & $20.7(174)$ \\
\hline Number of samples failed in-house $\mathrm{OC}<90 \%{ }^{\mathrm{j}}$ & 16 & $7.2(222)$ & NA & \\
\hline Number with STAT1:STAT3 $I_{50}$ ratio $\geq 2$-fold & 36 & $26.5(136)$ & 21 & $31.3(67)$ \\
\hline Number with promiscuous biological profile $(A F \geq 10 \%)^{k}$ & 5 & $13.9(36)$ & 3 & $14.3(21)$ \\
\hline STAT3-selective primary HCS actives & 31 & $22.8(136)$ & 18 & $26.9(67)$ \\
\hline
\end{tabular}

${ }^{a}$ In the $\%$ of total column, the numbers in brackets indicate the denominator used in the calculation.

${ }^{b} 728$ (Phases I and II, operation runs 1-8) and 279 (Phase III operation runs 9-15) active compounds inhibited IL-6-induced pSTAT3-Y705 activation $\geq 70 \%$ after a 3-h exposure at $20 \mu \mathrm{M}$ and were not either acutely cytotoxic and/or fluorescent outliers were ordered from the MLSCN SMR.

'An additional 304 compounds were ordered for phase III were selected by a 3D fragment-based Topomer similarity search method that used 13 phase I HCS priority hits as template compounds to search the 410,748 MLSCN compound library.

${ }^{\text {d} C h e r r y-p i c k ~ c o m p o u n d ~ o r d e r s ~ w e r e ~ s u b m i t t e d ~ t o ~ t h e ~ M L S C N ~ S M R ~ f o r ~} 728$ and 583 compounds for phases I and II and III, respectively.

${ }^{e}$ Only 668 and 519 of the requested samples for phases I and II and III, respectively, were available and delivered by the MLSCN SMR.

$f_{32}$ and 9 of the samples delivered for phases I and II and III, respectively, failed the SMR QC with $<90 \%$ purity.

${ }^{9}$ In phases I and II, the 668 samples were screened in triplicate wells at $20 \mu \mathrm{M}$ against IL-6-induced pSTAT3-Y705 activation and IFN $\gamma$-induced pSTAT1-Y701 activation after a 3-h compound exposure before cytokine activation; 33.2\% of compounds were confirmed active because they exhibited a mean \% inhibition of IL-6-induced PSTAT3 activation $\geq 70 \%$ at $20 \mu \mathrm{M}$.

${ }^{h}$ In phase III, the 519 samples were screened in triplicate wells at $20 \mu \mathrm{M}$ against IL-6-induced pSTAT3-Y705 activation after a 3-h compound exposure before 15-min cytokine activation; $33.3 \%$ of the compounds were confirmed active because they exhibited a mean \% inhibition of IL-6-induced pSTAT3 activation $\geq 50 \%$ at $20 \mu \mathrm{M}$.

'The confirmed actives from the screen were then confirmed in concentration-dependent pSTAT3 and PSTAT1 IC ${ }_{50}$ assays. Each compound was tested in a 10-point 2fold dilution series starting at a maximum concentration of $50 \mu \mathrm{M}$.

${ }^{\mathrm{j}}$ Confirmed actives from phases I and II were also subjected to in-house structural verification and LC/MS purity determinations.

${ }^{k}$ Cross target queries of the PubChem database were performed using the SID numbers of the concentration-dependent inhibitors of IL-6-induced pSTAT3 activation with $\mathrm{IC}_{50}$ s that were $\leq 25 \mu \mathrm{M}$ and were also $\geq 2$-fold lower than the corresponding IC ${ }_{50} \mathrm{~S}$ for IFN- $\gamma$-induced pSTAT3 activation. Any compound that was flagged active in $\geq 10 \%$ of the bioassays that it had been tested in $(\mathrm{AF} \geq 10 \%)$ was considered promiscuous and deprioritized.

NA, not applicable. 
inhibitors were then tested in 10-point 2-fold dilution series concentration-dependent pSTAT3 and pSTAT1 inhibition $\left(\mathrm{IC}_{50}\right)$ assays starting at a maximum concentration of $50 \mu \mathrm{M} ; 136(61.3 \%)$ of the compounds produced calculable $\mathrm{IC}_{50} \mathrm{~s}<50 \mu \mathrm{M}$ against IL-6-induced pSTAT3 activation, and 53 of these also exhibited $\mathrm{IC}_{50} \mathrm{~S}<50 \mu \mathrm{M}$ against IFN $\gamma$-induced pSTAT1 activation (Table 2). However, the remaining 83 concentration-dependent pSTAT3 activation inhibitors displayed evidence of selectivity and failed to produce calculable $\mathrm{IC}_{50} \mathrm{~s}$ against pSTAT1 activation at $<50 \mu \mathrm{M}$. An additional 16 samples failed our inhouse LC/MS quality control analysis because they were $<90 \%$ pure (Table 2). Thirty-six compounds with the following potency and STAT pathway selectivity criteria were prioritized for further consideration: compounds with pSTAT3 activation $\mathrm{IC}_{50} \mathrm{~s} \leq 25 \mu \mathrm{M}$ that were also $\geq 2$ fold lower than their corresponding $\mathrm{IC}_{50} \mathrm{~S}$ for pSTAT1 activation (Table 2 and Supplementary Table S1). A cross target query of the PubChem database was performed using the SID numbers of the 36 compounds and 5 were deprioritized for having a promiscuous biological profile because they had been flagged active in $>10 \%$ of the bioassays in which they were tested (Table 2). A total of 31 compounds from phases I and II of the HCS campaign met the following hit criteria: they achieved $\geq 70 \%$ inhibition of IL-6-induced pSTAT3 activation in the primary HCS assay and confirmation $(n=3)$ assays; they exhibited concentration-dependent inhibition of IL-6-induced pSTAT3 activation with $\mathrm{IC}_{50} \mathrm{~S} \leq 25 \mu \mathrm{M}$; they failed to produce calculable $\mathrm{IC}_{50} \mathrm{~S}$ against IFN $\gamma$-induced pSTAT1 activation at $<50 \mu \mathrm{M}$ or exhibited pSTAT1 activation $\mathrm{IC}_{50} \mathrm{~S}$ that were $\geq 2$-fold higher than the corresponding $\mathrm{IC}_{50} \mathrm{~S}$ for pSTAT3 activation; they were flagged inactive in $>90 \%$ of the assays in which they were tested in the PubChem database; and they were $\geq 90 \%$ pure by LC/MS analysis (Table 2 and Supplementary Table S1).

In phase III of the HCS campaign, 279 active compounds were identified that were not either acutely cytotoxic or fluorescent outliers (Table 2). An additional 304 compounds were requested for cherry-picks based on a Topomer similarity search of the MLSMR compound collection, minus the compounds screened in the primary HCS, using thirteen priority hits identified in phases I and II as template compounds. Only 519 of the 583 requested compounds were available for cherry-picking and 9 of the samples failed the SMR quality control criterion because they were $<90 \%$ pure (Table 2). In phase III, the cherry-picked samples were only confirmed in the IL-6-induced pSTAT3 activation assay at $20 \mu \mathrm{M}(n=3)$ before being selected for testing in concentration-dependent
pSTAT3 and pSTAT1 inhibition $\left(\mathrm{IC}_{50}\right)$ assays (Table 2). A total of 18 hit compounds from phase III of the HCS campaign achieved the STAT3 pathway inhibition potency, selectivity, and chemical purity criteria described above for the phase I and II hit confirmation (Table 2 and Supplementary Table S1). Five $(27.8 \%)$ of the phase III hits were from cherry-picked compounds selected by the Topomer similarity search of the MLSMR compound collection using phase I and II priority hit template compounds.

Characterization of Selective IL-6-Induced STAT3

Pathway Inhibitors

Since each of the 49 STAT3 pathway-selective hit compounds had, on average, been tested in $\sim 465$ bioassays in the PubChem database and had been flagged active in $<4 \%$ of these assays (Supplementary Table S1), they were considered reasonable starting points for further characterization. To select which of these hits should be considered for further analysis, we classified and clustered their chemical structures and used computational filters to identify and eliminate nuisance compounds and to predict their drug-like and ADME/ Tox properties. We also considered the chemical tractability of the hits. Based on this analysis, 26 compounds were purchased from commercial sources for additional characterization; 18 of these were classified and distributed among 5 clusters (Fig. $5 A$ and $B$ ), while the remaining 8 were considered as singleton hit structures (Fig. 5C). The amino alcohol cluster was the largest with 8 members (Fig. 5B), the guanidinoquinazoline and triazolothiadiazine clusters each contained 3 related structures, and the bis-thiazole and quinoline clusters both had 2 members (Fig. 5A).

These prioritized pSTAT3 pathway inhibitor hits were tested in 10-point 2-fold dilution series concentrationdependent pSTAT3 and pSTAT1 $\mathrm{IC}_{50}$ assays starting at a maximum concentration of $50 \mu \mathrm{M}$ (Fig. 6A-E and Table 3). They were also evaluated for their concentration-dependent ability to inhibit the growth of the Cal33, 686LN, FaDu, and OSC19 HNSCC cell lines after $72 \mathrm{~h}$ of compound exposure (Fig. $6 F$ and Table 3). Only 5 of the 26 compounds failed to exhibit $\geq 2$-fold selectivity for the inhibition of IL-6-induced pSTAT3 activation over IFN $\gamma$-induced pSTAT1 activation (Table 3); 2 amino alcohols (\# 12 and 15) and 3 singletons (\# 19, 23, and 26). The apparent pSTAT1:pSTAT3 $\mathrm{IC}_{50}$ ratios were $\geq 3$-fold for 13 of the remaining 21 compounds (Table 3). To illustrate the apparent pSTAT3 pathway selectivity of 5 representative hits, the concentration responses for inhibition of IL-6-induced pSTAT3 activation and IFN $\gamma$-induced pSTAT1 activation are presented (Fig. 6A-E). Compound 3, a member of the guanidinoquinazoline cluster, exhibited 


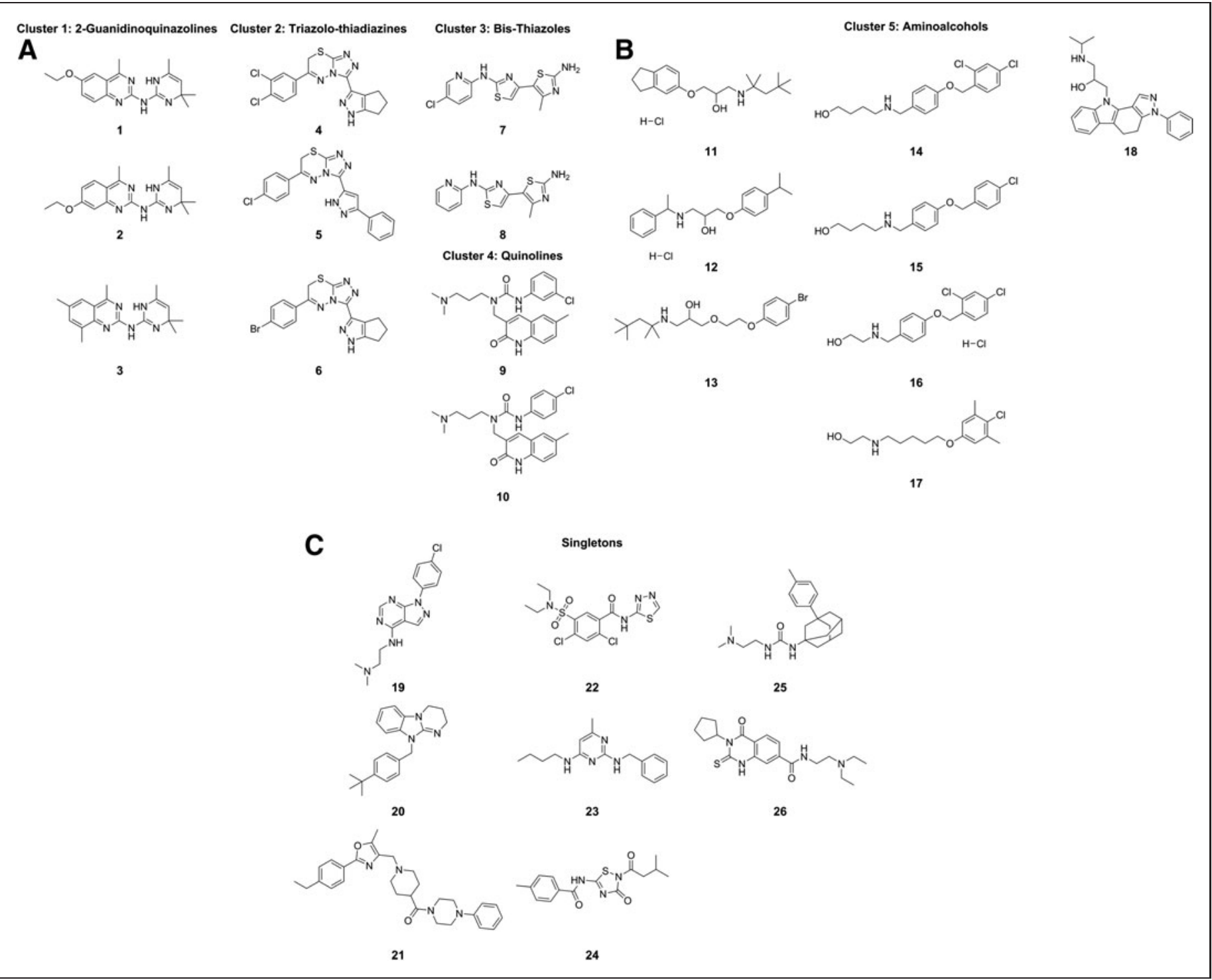

Fig. 5. Classification and clustering of the chemical structures of the 26 prioritized hits. (A) The guanidinoquinazoline $(n=3)$, triazolothiadiazine $(n=3)$, bis-thiazole $(n=2)$, and quinoline $(n=2)$ clusters. (B) The amino alcohol cluster $(n=8)$. (C) The singleton structures $(n=8)$. The compound numbers, $1-26$, of each structure correspond to those listed in column 2 of Table 3 , the hit characterization bioassay summary data, and the $\mathrm{IC}_{50}$ curves of selected compounds presented in Figure 6.

average $\mathrm{IC}_{50} \mathrm{~s}$ of $\sim 1$ and $6 \mu \mathrm{M}$ for inhibition of IL-6-induced pSTAT3 activation and IFN $\gamma$-induced pSTAT1 activation, respectively (Figs. 5A and $6 \mathrm{~A}$ and Table 3). Compound 4 from the triazolothiadiazine cluster displayed average $\mathrm{IC}_{50} \mathrm{~S}$ of $\sim 2$ and $48 \mu \mathrm{M}$ for inhibition of pSTAT3 and pSTAT1 activation, respectively (Figs. $5 A$ and $6 B$ and Table 3). The oxazole-piperazine singleton compound 21 produced average $\mathrm{IC}_{50} \mathrm{~S}$ of $\sim 0.5$ and $27 \mu \mathrm{M}$ for inhibition of pSTAT3 and pSTAT1 activation, respectively (Figs. 5C and 6C and Table 3). Two members of the amino alcohol cluster (Fig. 5C), compounds 11 and 18, exhibited average pSTAT1:pSTAT3 $\mathrm{IC}_{50}$ ratios of 4-fold and 3-fold, respectively (Fig. 6D, E, and Table 3). All 5 representative STAT3 pathway-selective hits inhibited the growth of Cal33 cells and 3 other HNSCC cell lines in a concentration-dependent manner (Fig. 5F and Table 3). Only 2 of the 26 prioritized hits failed to inhibit the growth of the 4 HNSCC cell lines at concentrations $\leq 50 \mu \mathrm{M}$ (Table 3), the bis-thiazole 7 and the singleton 24. The remaining 24 STAT3 pathway-selective hits inhibited the growth of all 4 HNSCC cell lines in a concentration- 


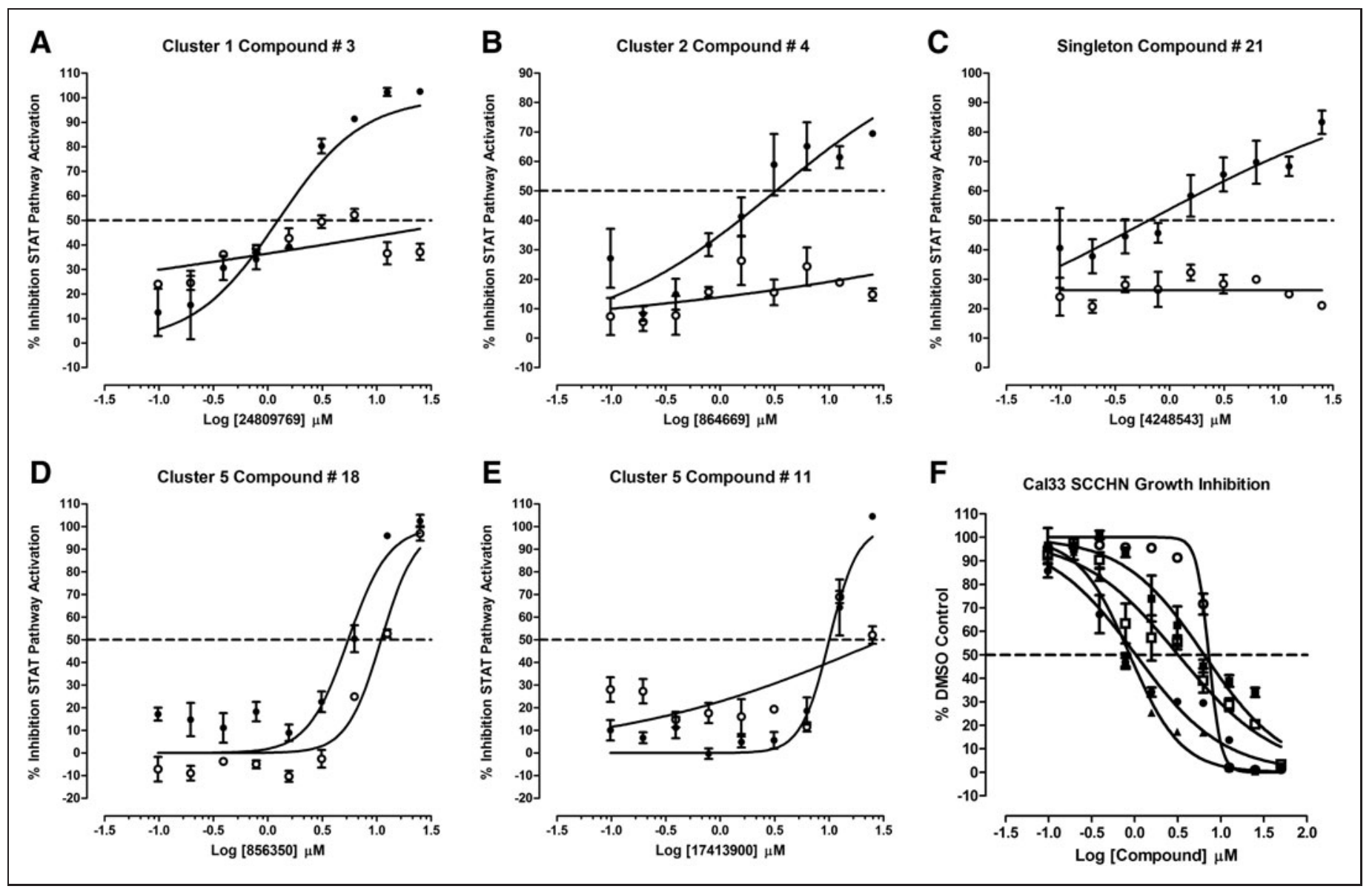

Fig. 6. Biological characterization of the 26 prioritized hits. (A) Concentration responses of compound \# 3 from the guanidinoquinazoline cluster in the IL-6-induced pSTAT3 activation and IFN $\gamma$-induced pSTAT1 activation assays. (B) Concentration responses of compound \# 4 from the triazolothiadiazine cluster in the IL-6-induced pSTAT3 activation and IFN $\gamma$-induced pSTAT1 activation assays. (C) Concentration responses of compound \# 21, the oxazole-piperazine singleton, in the IL-6-induced pSTAT3 activation and IFN $\gamma$-induced pSTAT1 activation assays. (D) Concentration responses of compound \# 18 from the amino alcohol cluster in the IL-6-induced pSTAT3 activation and IFN $\gamma$-induced pSTAT1 activation assays. (E) Concentration responses of compound \# 11 from the amino alcohol cluster in the IL-6-induced pSTAT3 activation and IFN $\gamma$-induced pSTAT1 activation assays. Three hundred eighty-four well plates were seeded with Cal33 HNSCC cells and serum starved for $24 \mathrm{~h}$ as described in the Materials and Methods section. After $24 \mathrm{~h}$, the serum starved Cal 33 cells were exposed to the indicated concentrations of the selected compounds for $3 \mathrm{~h}$ and then treated with either $50 \mathrm{ng} / \mathrm{mL}$ of IL-6 or $30 \mathrm{ng} / \mathrm{mL}$ IFN $\gamma$ for 15 min at $37^{\circ} \mathrm{C}$ in $5 \% \mathrm{CO}_{2}$ and $95 \%$ humidity and were then fixed and stained with Hoechst and either a mouse monoclonal anti-p-STAT3-Y705 antibody or a mouse monoclonal anti-pSTAT1-Y701 antibody as described in the Materials and Methods section. Images were acquired on the IXU and analyzed using the TE module as described in the Materials and Methods section. To calculate the \% inhibition of IL-6-induced pSTAT3 activation, the mean average inner pSTAT3-Y705 intensity values of the $0.2 \%$ DMSO Min plate control wells $(n=32)$ and the $50 \mathrm{ng} / \mathrm{mL}$ IL- 6 Max plate control wells $(n=32)$ were used to normalize the mean average inner intensity pSTAT3-Y705 values of the compound-treated wells and to represent $100 \%$ and $0 \%$ inhibition of IL-6-induced PSTAT3 activation, respectively. To calculate the \% inhibition of IFN $\gamma$-induced pSTAT1 activation, the mean average inner pSTAT1-Y701 intensity values of the $0.2 \%$ DMSO Min plate control wells $(n=32)$ and the $30 \mathrm{ng} / \mathrm{mL}$ IFN $\gamma$ Max plate control wells $(n=32)$ were used to normalize the mean average inner intensity PSTAT1-Y701 values of the compound-treated wells and to represent $100 \%$ and $0 \%$ inhibition of IFN $\gamma$-induced pSTAT1 activation, respectively. The mean \pm SD $(n=3) \%$ inhibition of IL-6-induced pSTAT3 activation (O) and the mean \pm SD $(n=3) \%$ inhibition of IFN $\gamma$-induced pSTAT1 activation $(\square)$ from triplicate wells at the indicated compound concentrations are presented. Representative experimental data from one of 2 independent experiments are shown. (F) Cal33 HNSCC growth inhibition for compound \#s 3, 4, 11, 18, and 21; 1000 Cal33 $\mathrm{HNSCC}$ cells were seeded into 384 -well assay plates and incubated at $37^{\circ} \mathrm{C}$ in $5 \% \mathrm{CO}_{2}$ and $95 \%$ humidity for $24 \mathrm{~h}$. After $24 \mathrm{~h}$, the indicated concentrations of test compounds were transferred into the test wells of the 384 -well assay plates that were then incubated at $37^{\circ} \mathrm{C}$ in $5 \% \mathrm{CO}_{2}$ and $95 \%$ humidity for an additional $72 \mathrm{~h}$. Control wells received DMSO alone. After $72 \mathrm{~h}$ of exposure to the compounds, CellTiter-Glo detection reagent was dispensed into the wells of the assay plates, and the luminescent signal was captured on the Envision microtiter plate reader. The mean \pm SD $(n=3)$ growth inhibition data from triplicate wells for each compound concentration are presented as the \% of the DMSO plate controls. Compound \# 3 from the guanidinoquinazoline cluster ( $)$; compound \# 11 from the amino alcohol cluster $(\bigcirc)$; compound \# 4 from the triazolothiadiazine cluster $(\square)$; compound \# 21, the oxazole-piperazine singleton $(\boldsymbol{\square})$; compound \# 18 from the amino alcohol cluster $(\boldsymbol{\Delta})$. Representative experimental data from one of 2 independent experiments are shown. 


\begin{tabular}{|c|c|c|c|c|c|c|c|c|c|c|c|c|c|c|}
\hline \multirow{2}{*}{\multicolumn{3}{|c|}{$\begin{array}{l}\text { Purchased compound } \\
\text { Information }\end{array}$}} & \multicolumn{4}{|c|}{ STAT HCS assay inhibition } & \multicolumn{8}{|c|}{ Head and neck squamous cell carcinoma growth inhibition } \\
\hline & & & \multicolumn{2}{|c|}{$\begin{array}{c}\text { pSTAT3-Y705 } \\
\text { IC }_{50} \mu \mathrm{M}\end{array}$} & \multicolumn{2}{|c|}{$\begin{array}{c}\text { pSTAT1-Y701 } \\
\text { IC }_{50} \mu \mathrm{M}\end{array}$} & \multicolumn{2}{|c|}{$\begin{array}{c}\text { Cal33 } \\
I_{50} \mu \mathrm{M}\end{array}$} & \multicolumn{2}{|c|}{$\begin{array}{c}\text { 686LN } \\
I_{50} \mu \mathrm{M}\end{array}$} & \multicolumn{2}{|c|}{$\begin{array}{c}\text { FADU } \\
I C_{50} \mu \mathrm{M}\end{array}$} & \multicolumn{2}{|c|}{$\begin{array}{l}\text { OSC19 } \\
I_{50} \mu M\end{array}$} \\
\hline SID & $\#$ & Source & Mean & SDM & Mean & SDM & Mean & SDM & Mean & SDM & Mean & SDM & Mean & SDM \\
\hline 16953031 & 1 & $\mathrm{HCS}-1^{\mathrm{a}}$ & 8.841 & 5.156 & $>50^{b}$ & NA & 19.855 & 9.88 & 18.170 & 3.14 & 13.179 & 8.28 & 15.350 & 1.40 \\
\hline 857319 & 2 & HTS- $2^{c}$ & 20.666 & 3.023 & $>50$ & NA & 27.538 & 12.356 & 26.510 & 8.075 & 21.040 & 4.036 & 10.857 & 3.727 \\
\hline 24809769 & 3 & SSS $^{d} 16953031$ & 0.790 & 0.637 & 5.935 & 0.933 & 1.242 & 0.300 & 2.427 & 0.309 & 1.957 & 0.080 & 3.250 & 0.375 \\
\hline 864669 & 4 & HCS-1 & 1.841 & 1.920 & 47.598 & $n=1^{e}$ & 9.040 & 8.30 & 12.072 & 5.84 & 10.228 & 5.49 & 24.010 & 6.86 \\
\hline 17401700 & 5 & SSS 864669 & 6.604 & 1.781 & 43.656 & $n=1$ & 22.098 & 3.405 & 14.625 & 0.864 & 14.533 & 0.041 & 20.144 & 2.416 \\
\hline 87346004 & 6 & SSS 864669 & 14.052 & 12.574 & $>50$ & NA & $<0.1$ & NA & 0.458 & 0.132 & 0.029 & NA & 1.803 & 0.091 \\
\hline 4261142 & 7 & HCS-1 & 3.751 & 4.042 & 16.701 & $n=1$ & $>50$ & NA & 43.150 & 3.39 & $>50$ & NA & $>50$ & NA \\
\hline 7974614 & 8 & SSS 4261142 & 13.791 & 3.189 & 36.038 & $n=1$ & 6.809 & 2.609 & 26.048 & 1.390 & 14.823 & 0.114 & 15.958 & 0.954 \\
\hline 16953265 & 9 & HCS-1 & 10.953 & 12.770 & 22.243 & 13.755 & 8.794 & 3.84 & 27.785 & 3.60 & 13.420 & 0.41 & 17.590 & 2.18 \\
\hline 14722673 & 10 & HTS-2 & 10.984 & 4.905 & 31.363 & 7.886 & 4.751 & 1.597 & 10.865 & 0.631 & 17.744 & 0.696 & 13.484 & 0.799 \\
\hline 17413900 & 11 & HCS-1 & 5.299 & 6.730 & 22.420 & $n=1$ & 8.270 & 1.94 & 19.645 & 12.32 & 4.427 & 0.08 & 8.155 & 0.71 \\
\hline 49673704 & 12 & SSS 1743900 & 11.578 & 2.599 & 15.533 & 3.586 & 15.009 & 0.832 & 14.883 & 0.395 & 9.759 & 3.477 & 13.260 & 1.491 \\
\hline 22404614 & 13 & SSS 1743900 & 14.018 & 8.149 & $>50$ & NA & 27.983 & 0.587 & 17.310 & 2.092 & 41.001 & 1.610 & 39.498 & 0.492 \\
\hline 17387568 & 14 & HCS-1 & 6.525 & 0.983 & 23.335 & 5.789 & 6.579 & 0.43 & 22.800 & 1.90 & 11.330 & 1.23 & 7.731 & 1.31 \\
\hline 17387569 & 15 & HTS-2 & 22.751 & 12.725 & 29.082 & $n=1$ & 17.607 & 6.963 & 35.463 & 3.629 & 28.780 & 2.271 & 18.819 & 4.402 \\
\hline 24793247 & 16 & SSS 17387568 & 7.530 & 2.491 & 20.999 & 6.730 & 4.409 & 0.796 & 21.087 & 2.550 & 7.973 & 0.205 & 11.126 & 0.413 \\
\hline 7975387 & 17 & SSS 17387568 & 15.554 & 4.671 & 37.772 & 9.331 & 5.204 & 1.350 & 29.848 & 0.512 & 14.747 & 0.502 & 17.924 & 0.753 \\
\hline 856350 & 18 & HTS-2 & 4.423 & 1.516 & 13.771 & 3.903 & 0.646 & 0.323 & 3.343 & 0.128 & 2.020 & 0.005 & 7.096 & 0.405 \\
\hline 7971198 & 19 & HTS-2 & 18.538 & 3.207 & 23.672 & 3.285 & 7.229 & 2.402 & 27.474 & 1.694 & 27.930 & 0.733 & 16.722 & 0.789 \\
\hline 14721602 & 20 & HCS-1 & 12.252 & 11.431 & $>50$ & NA & 3.402 & 0.83 & 6.745 & 0.62 & 5.941 & 1.12 & 4.580 & 0.14 \\
\hline 4248543 & 21 & HTS-2 & 0.500 & 0.176 & 26.928 & $n=1$ & 5.176 & 0.198 & 2.044 & 0.345 & 11.225 & 2.680 & 10.011 & 2.088 \\
\hline 4264603 & 22 & HCS-1 & 5.148 & 5.557 & 26.460 & 15.993 & 33.935 & 2.09 & 14.845 & 0.78 & 14.025 & 4.72 & 26.595 & 1.68 \\
\hline 858611 & 23 & HTS-2 & 9.452 & 4.077 & 16.681 & 3.464 & 1.716 & 0.177 & 4.221 & 0.405 & 1.507 & 0.259 & 6.086 & 0.707 \\
\hline 17410825 & 24 & HTS-2 & 9.809 & 5.445 & 26.617 & 1.772 & $>50$ & NA & $>50$ & NA & $>50$ & NA & $>50$ & NA \\
\hline 16952763 & 25 & HCS-1 & 10.281 & 6.457 & 27.753 & 3.366 & 16.175 & 2.30 & 32.630 & 3.79 & 20.170 & 0.48 & 22.945 & 3.22 \\
\hline 14737707 & 26 & HCS-1 & 14.483 & 17.492 & 10.684 & $n=1$ & 12.576 & 5.65 & 38.890 & 13.44 & 24.100 & 0.08 & 14.335 & 1.53 \\
\hline
\end{tabular}

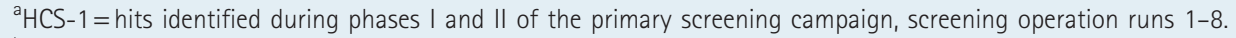

${ }^{b}>50$ indicates the compound failed to achieve $\geq 50 \%$ inhibition at the maximum concentration tested in both of the replicate $I_{50}$ experiments.

${ }^{\mathrm{C}} \mathrm{HCS}-2$ = hits identified during phase III of the primary screening campaign, screening operation runs 9-15.

${ }^{\mathrm{d} S S S}=$ hits identified from the similarity and substructure search cherry-pick order selected from the MLSCN SMR library based on HCS-1 hits.

${ }^{e} n=1$ indicates that only 1 of the 2 replicate curve fits produced a calculable $\mathrm{IC}_{50}$, which is reported, and the other did not achieve $\geq 50 \%$ inhibition at the maximum concentration tested $=>50$.

NA, not applicable.
} 
dependent manner; 6 exhibited average HNSCC growth inhibition $\mathrm{IC}_{50} \mathrm{~S}$ in the $1-10 \mu \mathrm{M}$ range, 12 produced average $\mathrm{IC}_{50} \mathrm{~S}$ in the $10-20 \mu \mathrm{M}$ range, and 6 displayed average $\mathrm{IC}_{50} \mathrm{~S}$ in the $20-32 \mu \mathrm{M}$ range (Table 3).

\section{DISCUSSION}

There is a growing body of preclinical and clinical evidence that IL-6 production by HNSCC cells and/or stromal cells and infiltrating immune cell populations in the tumor microenvironment contribute to HNSCC progression and drug resistance. ${ }^{26,27,31,32,36,54-60}$ Elevated concentrations of IL-6 were found in the saliva of patients with premalignant HNSCC lesions. ${ }^{26}$ Higher pretreatment serum IL-6 levels were associated with lower HNSCC overall survival, higher tumor recurrence, and increased development of second primary tumors. $^{26,31,36,59}$ In patient HNSCC tumor tissue specimens, IL-6 levels correlated with the HNSCC tumor grade and were reduced after effective radio- or chemotherapies. ${ }^{55,57} \mathrm{IL}-6 \mathrm{R} \alpha$ mRNA levels were higher in oral cancer cells relative to normal mucosa, and increased IL-6R $\alpha$ expression was associated with larger tumors of advanced histological stage. ${ }^{55,57}$ Elevated pSTAT3 and IL-6 staining in HNSCC patient tumor specimens were significantly linked with lower response rates and shorter survival times for patients that received surgical resection combined with adjuvant radiotherapy, with or without chemotherapy. ${ }^{26,55,57,59}$ Low baseline serum IL-6 and VEGF levels were identified as potential prognostic markers for cetuximab-containing therapies. ${ }^{26,55,57}$ Several studies have indicated that IL-6 induces EMT in HNSCC cell lines and promotes their ability to invade and metastasize. $^{32,55,58,60}$ For example, IL-6 promoted HNSCC metastasis to the lymph nodes and lungs in an SCID mouse xenograft model by inducing EMT through the JAK-STAT3-SNAIL signaling pathway. ${ }^{60}$ IL-6 treatment of HNSCC cell lines induced the hypermethylation and silencing of several tumor suppressors (CHFR, GATA5, PAX6, and SOCS3), thereby promoting HNSCC tumorigenesis. ${ }^{56}$ Exogenous IL-6 treatment or intrinsic IL-6 signaling enhanced the resistance of HNSCC cell lines to irradiation and EGFR inhibitors. ${ }^{55}$ The IL-6neutralizing MAb tocilizumab inhibited HNSCC cell line growth and VEGF expression in vitro, dramatically reduced tumor cell pSTAT3 expression in mice, and suppressed tumor angiogenesis and in vivo HNSCC growth in HNSCC xenograft models. ${ }^{57}$ Several studies have indicated that HNSCC cell lines synthesize and secrete IL-6. ${ }^{26,27,32,59}$ In one study, pSTAT3-Y705 levels were higher in the majority of HNSCC cell lines tested, although only $3 / 10$ cell lines exhibited activated EGFR and pSTAT3 persisted after treatment with the EGFR inhibitor, AG1478. ${ }^{27}$ HNSCC cell line conditioned medium (CM) induced pSTAT3-Y705 activation independently of EGFR signaling, and blocking gp130 activity with a neutralizing antibody abolished HNSCC CM-induced pSTAT3-Y705 activation. ${ }^{27}$ Treatment of HNSCC cell lines with the JAK inhibitor, AG490, reduced pSTAT3-Y705 levels and caused significant growth inhibition and apoptosis. ${ }^{27}$ It has been proposed that constitutive activation of STAT3 in HNSCC through the autocrine and/or paracrine effects of IL-6 conferred a proliferative and survival advantage that may be responsible for the lack of efficacy of EGFR-targeted therapies. ${ }^{27,55}$

We screened 94,491 compounds from the MLSCN library at $20 \mu \mathrm{M}$ for their ability to inhibit IL-6-induced pSTAT3 activation in Cal33 HNSCC cells (Figs. 1-3 and Table 1). For contractual reasons, the primary HCS was conducted over several months in 3 distinct phases, each separated by 1 month; the first screening run was completed in phase I, runs 2-8 were conducted in phase II, and runs 9-15 were completed in phase III. After the QC review of operation runs 4 and 5 flagged some failed plates (Fig. 3), we conducted additional troubleshooting studies and amended the HCS protocol to ensure that only Cal33 cells with passage \#s $<20$ and reconstituted IL- 6 that had been stored at $-80^{\circ} \mathrm{C}$ for $\leq 4$ weeks would be utilized (Fig. 4). Despite the intermittent implementation, the IL-6-induced pSTAT3 activation imaging assay performed very well in a high-throughput manner, and $<4 \%$ of the 294 assay plates failed and were retested (Fig. 3). After cytotoxic and fluorescent outliers were identified and removed (Supplementary Figs. S1 and S2), 1,068 (1.1\%) primary HCS actives that inhibited IL-6-induced pSTAT3 activation by $\geq 70 \%$ remained (Table 1).

A total of 1,187 compounds were cherry-picked for confirmation; these consisted of the available actives identified in the primary HCS and additional compounds that were selected from a similarity search of the remaining MLSCN library using selected hit structures identified in phases I and II of the screen; 394 (33.2\%) compounds reproducibly inhibited IL-6induced pSTAT3 activation by $\geq 70 \%$ at $20 \mu \mathrm{M}$ and 203 (51.5\%) of these were confirmed as concentration-dependent inhibitors with $\mathrm{IC}_{50} \mathrm{~S} \leq 50 \mu \mathrm{M}$ (Table 2). Activation of the STAT3 signaling pathway initiates transcriptional activation of target genes that inhibit apoptosis, promote cell proliferation and survival, stimulate angiogenesis and metastasis, and hinder antitumor immune responses. ${ }^{1-4,7,61}$ In contrast, activation of the STAT1 signaling pathway leads to the transcriptional activation of genes that activate cell cycle arrest, promote apoptosis, and enhance antitumor immunity. ${ }^{61-63}$ Although STAT3 and STAT1 are highly homologous and interact with very similar DNA-binding sites, in many 
physiological situations, they are regulated in a reciprocal manner to produce opposing effects. ${ }^{4,61,62}$ For example, in cancer cells, activated STAT3 behaves as an oncogene, while activated STAT1 functions as a tumor suppressor. ${ }^{4,61,62}$ It is our hypothesis that small molecules that selectively inhibit STAT3 pathway activation without significantly blocking STAT1 signaling might have a suitable bioactivity profile for anticancer drug development. ${ }^{39}$ We therefore implemented the IFN $\gamma$-induced pSTAT1 activation assay to prioritize hits that selectively inhibited the oncogenic effects of STAT3 activation over the tumor suppressor functions of activated STAT1; 89 (43.8\%) of the pSTAT3 inhibitor hits also produced $\mathrm{IC}_{50} \mathrm{~S}$ against IFN- $\gamma$-induced pSTAT1 activation. Forty-nine compounds met the following hit criteria: they were $\geq 90 \%$ pure; at $20 \mu \mathrm{M}$, they exhibited $\geq 70 \%$ inhibition of pSTAT3 activation in HCS and confirmation assays; their pSTAT3 activation $\mathrm{IC}_{50} \mathrm{~s}$ were $\leq 25 \mu \mathrm{M}$; they were $\geq 2$-fold selective for pSTAT3 inhibition over pSTAT1 inhibition; and a cross target query of PubChem indicated they were not biologically promiscuous (Supplementary Table S1).

To select which of these hits should be further characterized, we classified and clustered their chemical structures and applied computational filters (PAINS/REOS) ${ }^{64,65}$ to identify and eliminate nuisance compounds and to predict their druglike and ADME/Tox properties. Furthermore, we also considered their synthetic tractability. The chemical structures of the 26 selected hits were distributed among 5 clusters and 8 singletons (Fig. 5). Twenty-four of the 26 compounds produced $\mathrm{IC}_{50} \mathrm{~s} \leq 20 \mu \mathrm{M}$ for inhibition of IL-6-induced pSTAT3 activation, 21 of these were $\geq 2$-fold selective for inhibition of pSTAT 3 versus pSTAT 1 , and 13 achieved a more rigorous $\geq 3$ fold selectivity criterion (Fig. 6 and Table 3). Twenty-four of the 26 compounds inhibited the growth of all 4 HNSCC cell lines, with 18 of the hits producing average $\mathrm{IC}_{50} \mathrm{~s} \leq 20 \mu \mathrm{M}$ (Fig. 6 and Table 3).

In classical IL-6 signal transduction, IL- 6 binds to IL- 6 receptor alpha subunits (IL-6R $\alpha$, gp80) on cells that express this receptor and this binary complex then recruits a gp130 signaling subunit to form an IL-6/IL-6R $\alpha / g p 130$ heterotrimer, and then 2 of these interact to form a hexamer-activated IL-6/ IL-6R $\alpha / g p 130$ signaling complex. ${ }^{21,22,66}$ Homodimerization of the IL-6/IL-6R $\alpha / g p 130$ heterotrimers triggers phosphorylation of the gp130-associated Janus kinase, and subsequent phosphorylation of tyrosine residues on the intracellular cytoplasmic tail of gp130 generates docking sites for the SH2 domains of STAT3. ${ }^{21,22,24,66}$ JAK-mediated phosphorylation of the tyrosine-705 residue of STAT3 recruited to the activated

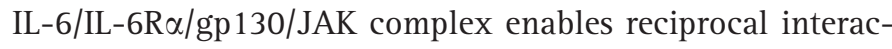
tions between $\mathrm{SH} 2$ domains of pSTAT3 monomers to form dimers, which then translocate into the nucleus. ${ }^{4,21,22,24,66}$ In the nucleus, pSTAT3 dimers bind to specific DNA response elements in the promoters that activate the transcription of target genes that favor the development, progression, and maintenance of HNSCC and many other tumors. ${ }^{1-4,7}$ However, IL- 6 can also activate signaling in tissues and cells that do not express the IL- 6 R $\alpha$ receptor by a trans-signaling process. ${ }^{38} \mathrm{IL}-$ 6 binds to a soluble form of the IL-6R $\alpha$ (sIL-6R $\alpha$ ) that is formed either by the expression of an alternatively spliced IL-6R $\alpha$ mRNA variant or by the shedding of the membrane form of IL$6 \mathrm{R} \alpha$ after limited proteolysis by a variety of matrix metalloproteases (MMPs), including ADAM17. ${ }^{26,38}$ The IL-6/sIL-6R $\alpha$ complex can bind to gp130 subunits on cells, which do not express the IL-6R $\alpha$ to activate downstream signaling pathways. ${ }^{26,38}$ In cancer, altered proteolysis leads to unregulated tumor growth, tissue remodeling, inflammation, tissue invasion, and metastasis. ${ }^{67}$ MMPs are proteolytic enzymes capable of cleaving extracellular matrix components, growth factors, and cell surface receptors that play important roles during cancer progression, including tumor angiogenesis, invasion, and metastasis. ${ }^{68}$ Studies have demonstrated that HNSCC expresses high levels of MMPs in vivo and that inhibition of these enzymes in vitro and in mouse models decreases HNSCC invasion and metastasis. ${ }^{69}$ In addition, many HNSCC tumorassociated stromal cells (fibroblasts, endothelial, and inflammatory cells) synthesize and secret MMPs. ${ }^{68,69}$ IL-6 produced and secreted either by HNSCC cells and/or the associated stromal cells within the tumor will activate the STAT3 signaling pathway in cells that express the IL-6R $\alpha$ receptor through an autocrine or paracrine manner. For cells in the HNSCC tumor that do not express the IL-6R $\alpha$ receptor, the combination of elevated IL- 6 levels and sIL-6R $\alpha$ released by MMPs in the tumor microenvironment can activate the STAT3 signaling pathway by trans-signaling.

Preclinical and clinical studies have implicated elevated IL-6 levels in the HNSCC tumor microenvironment with activation of the STAT3 signaling pathway, tumor progression, and drug resistance. ${ }^{26,27,31,32,36,54-60}$ Monoclonal antibodies that target either IL- 6 or IL-6R $\alpha$ have been approved for the treatment of inflammatory diseases and are in ongoing clinical trials for multiple myeloma, prostate cancer, and metastatic kidney cancer. ${ }^{35,38}$ Currently, there are no smallmolecule inhibitors in clinical development that target the activation of the IL-6/IL-6R $\alpha /$ gp 130/JAK complex. We have successfully implemented an HCS campaign that identified several hit series that selectively blocked IL-6-induced STAT3 activation over IFN- $\gamma$-induced STAT1 activation and inhibited the growth of HNSCC cells in vitro. Four chemical series were considered suitable starting points for lead 
optimization: the guanidinoquinazolines, ${ }^{70}$ the triazolothiadiazines, the amino alcohols, and an oxazole-piperazine singleton. The triazolothiadiazine and oxazole-piperazine lead series have been progressed through hit-to-lead activities that will be reported elsewhere. In vitro mechanism of action studies indicate that these compounds do not inhibit IL-6 receptor binding and are not kinase inhibitors, but rather

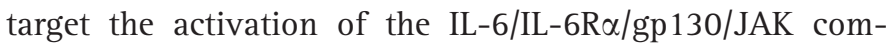
plex (manuscript in preparation). Optimization of the triazolothiadiazine series generated analogs appropriate for in vivo pharmacokinetic and maximum tolerated dose determinations in mice in preparation for in vivo efficacy testing in our established Cal33 HNSCC mouse xenograft model. Information with patient-derived xenografts would also be useful.

\section{ACKNOWLEDGMENTS}

The authors would like to thank Mr. Pete Chambers for LCMS/ELS analyses and Ms. Mary Liang for sample handling and procurement, as well as data handling and processing. This project has been funded, in part, with Federal Funds from the National Cancer Institute, National Institutes of Health, under Contract No. HSN261200800001E. The content of this publication does not necessarily reflect the views or policies of the Department of Health and Human Services, nor does mention of trade names, commercial products, or organizations imply endorsement by the U.S. Government. This work was supported by the NExT-CBC Project ID \#1015, S08-221 Task Order 6 "STAT3 Pathway Inhibitor HCS" (Grandis, PI), NCI Chemical Biology Consortium, Pittsburgh Specialized Application Center (PSAC) (Lazo and Johnston co-PIs), and University of Pittsburgh Chemical Diversity Center (Huryn, PI). The project was also supported, in part, by funds from the American Cancer Society (Grandis) and a Head and Neck Spore P50 award (Grandis, CA097190).

\section{DISCLOSURE STATEMENT}

No competing financial interests exist.

\section{REFERENCES}

1. Frank DA: STAT3 as a central mediator of neoplastic cellular transformation. Cancer Lett 2007;251:199-210.

2. Germain D, Frank DA: Targeting the cytoplasmic and nuclear functions of signal transducers and activators of transcription 3 for cancer therapy. Clin Cancer Res 2007;13:5665-5669.

3. Jing N, Tweardy DJ: Targeting Stat3 in cancer therapy. Anticancer Drugs 2005; 16:601-607.

4. Johnston PA, Grandis JR: STAT3 signaling: anticancer strategies and challenges. Mol Interv 2011;11:18-26.

5. Quesnelle KM, Boehm AL, Grandis JR: STAT-mediated EGFR signaling in cancer. J Cell Biochem 2007;102:311-319.
6. Aggarwal $B B$, Kunnumakkara $A B$, Harikumar $K B$, et al.: Signal transducer and activator of transcription-3, inflammation, and cancer: how intimate is the relationship? Ann N Y Acad Sci 2009;1171:59-79.

7. Leeman RJ, Lui VW, Grandis JR: STAT3 as a therapeutic target in head and neck cancer. Expert Opin Biol Ther 2006;6:231-241.

8. Seethala RR, Gooding WE, Handler PN, et al:: Immunohistochemical analysis of phosphotyrosine signal transducer and activator of transcription 3 and epidermal growth factor receptor autocrine signaling pathways in head and neck cancers and metastatic lymph nodes. Clin Cancer Res 2008;14:13031309.

9. Boehm $A L$, Sen $M$, Seethala $R$, et al:: Combined targeting of epidermal growth factor receptor, signal transducer and activator of transcription-3, and $\mathrm{BCl}-\mathrm{X}(\mathrm{L})$ enhances antitumor effects in squamous cell carcinoma of the head and neck. Mol Pharmacol 2008;73:1632-1642.

10. Egloff AM, Grandis JR: Improving response rates to EGFR-targeted therapies for head and neck squamous cell carcinoma: candidate predictive biomarkers and combination treatment with Src inhibitors. J Oncol 2009;2009:896407.

11. Leeman-Neill RJ, Wheeler SE, Singh SV, et al.: Guggulsterone enhances head and neck cancer therapies via inhibition of signal transducer and activator of transcription-3. Carcinogenesis 2009;30:1848-1856.

12. Brockstein B. Management of recurrent head and neck cancer: recent progress and future directions. Drugs 2011;71:1551-1559.

13. Goerner M, Seiwert TY, Sudhoff H: Molecular targeted therapies in head and neck cancer-an update of recent developments. Head Neck Oncol 2010;2:8-12.

14. Stransky N, Egloff AM, Tward AD, et al:: The mutational landscape of head and neck squamous cell carcinoma. Science 2012;333:1157-1160.

15. Ramqvist $T$, Dalianis $T$ : Oropharyngeal cancer epidemic and human papillomavirus. Emerg Infect Dis 2010;16:1671-1677.

16. Denaro N, Russi EG, Adamo V, Colantonio I, Merlano MC: Postoperative therapy in head and neck cancer: state of the art, risk subset, prognosis and unsolved questions. Oncology 2011;81:21-29.

17. Li H, Wawrose JS, Gooding WE, Garraway LA, Lui VW, Peyser ND, Grandis JR: Genomic analysis of head and neck squamous cell carcinoma cell lines and human tumors: a rational approach to preclinical model selection. Mol Cancer Res 2014;12:571-582.

18. Perez-Ordonez B, Beauchemin M, Jordan RCK: Molecular biology of squamous cell carcinoma of the head and neck. J Clin Pathol 2006;59:445-453.

19. Koskela H, Eldfors $S$, Ellonen $P$, et al.: Somatic STAT3 mutations in large granular lymphocytic leukemia. N Engl J Med 2012;366:1905-1913.

20. Rajala $H_{1}$ Olson $T$, Clemente MJ, et al.: The analysis of clonal diversity and therapy responses using STAT3 mutations as a molecular marker in large granular lymphocytic leukemia. Haematologica 2015;100:91-99.

21. Heinrich PC, Behrmann I, Müller-Newen G, Schaper F, Graeve L: Interleukin-6type cytokine signalling through the gp130/Jak/STAT pathway. Biochem J 1998;334:297-314.

22. Heinrich PC, Behrmann I, Haan S, Hermanns HM, Müller-Newen G, Schaper F: Principles of interleukin (IL)-6-type cytokine signalling and its regulation. Biochem J 2003;374:1-20.

23. Ram PT, lyengar R: G protein coupled receptor signaling through the Src and Stat3 pathway: role in proliferation and transformation. Oncogene 2001;20: 1601-1606.

24. Wilks AF: The JAK kinases; Not just another kinase drug discovery target. Semin Cell Dev Biol 2008;19:319-328.

25. Chen LF, Cohen EE, Grandis JR: New strategies in head and neck cancer: understanding resistance to epidermal growth factor receptor inhibitors. Clin Cancer Res 2010;16:2489-2495.

26. Culig Z: Interleukin-6 as a therapy target in oral squamous cell carcinoma. Expert Opin Ther Targets 2013;17:53-59.

27. Sriuranpong V, Park JI, Amornphimoltham P, Patel V, Nelkin BD, Gutkind JS: Epidermal growth factor receptor-independent constitutive activation of STAT3 in head and neck squamous cell carcinoma is mediated by the autocrine/ paracrine stimulation of the interleukin 6/gp130 cytokine system. Cancer Res 2003;63:2948-2956. 
28. Ara T, Nakata R, Sheard MA, et al:: Critical role of STAT3 in IL-6-mediated drug resistance in human neuroblastoma. Cancer Res 2013;73:3852-3864.

29. Brocke-Heidrich $K$, Kretzschmar AK, Pfeifer $G$, et al.: Interleukin-6-dependent gene expression profiles in multiple myeloma INA-6 cells reveal a Bcl-2 familyindependent survival pathway closely associated with Stat3 activation. Blood 2004;103:242-251.

30. Culig Z, Puhr M: Interleukin-6: A multifunctional targetable cytokine in human prostate cancer. Mol Cell Endocrinol 2012;360:52-58.

31. Duffy S, Taylor JM, Terrell JE, et al.: Interleukin-6 predicts recurrence and survival among head and neck cancer patients. Cancer 2008;113: 750-757.

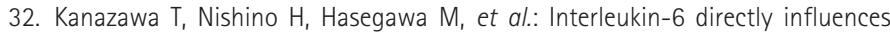
proliferation and invasion potential of head and neck cancer cells. Eur Arch Otorhinolaryngol 2007;264:815-821.

33. Karkera J, Steiner $H_{1}$ Li W, et al:: The anti-interleukin-6 antibody siltuximab down-regulates genes implicated in tumorigenesis in prostate cancer patients from a phase I study. Prostate 2011;71:1455-1465.

34. Stark J, Li H, Kraft P, et al.: Circulating prediagnostic interleukin-6 and Creactive protein and prostate cancer incidence and mortality. Int J Cancer 2009; 124:2683-2689.

35. Hong $D$, Angelo LS, Kurzrock R: Interleukin-6 and its receptor in cancer: implications for translational therapeutics. Cancer 2007;110:1911-1928.

36. Duffy $S$, Teknos T, Taylor JM, et al.: Health behaviors predict higher interleukin-6 levels among patients newly diagnosed with head and neck squamous cell carcinoma. Cancer Epidemiol Biomarkers Prev 2013;22:374-381.

37. Shetty $A$, Hanson $R$, Korsten $P$, et al:: Tocilizumab in the treatment of rheumatoid arthritis and beyond. Drug Des Devel Ther 2014;28:349-364.

38. Jones S, Scheller J, Rose-John S: Therapeutic strategies for the clinical blockade of IL-6/gp130 signaling. J Clin Invest 2011;121:3375-3383.

39. Johnston $P$, Sen $M$, Hua $Y$, et al:: High-content pSTAT3/1 imaging assays to screen for selective inhibitors of STAT3 pathway activation in head and neck cancer cell lines. Assay Drug Dev Technol 2014;12:55-79.

40. Bauer V, Hieber L, Schaeffner Q, et al.: Establishment and Molecular Cytogenetic Characterization of a Cell Culture Model of Head and Neck Squamous Cell Carcinoma (HNSCC). Genes 2010;1:338-412.

41. Gioanni J, Fischel JL, Lambert JC, et al.: Two new human tumor cell lines derived from squamous cell carcinomas of the tongue: establishment, characterization and response to cytotoxic treatment. Eur J Concer Clin Oncol 1988;24:14451455.

42. Khan $M$, Yasuda $M$, Higashino $F$, et al.: nm23-H1 suppresses invasion of oral squamous cell carcinoma-derived cell lines without modifying matrix metalloproteinase-2 and matrix metalloproteinase-9 expression. Am J Pathol 2001;158:1785-1791.

43. Rangan S: A new human cell line ( $\mathrm{FaDu})$ from a hypopharyngeal carcinoma. Cancer 1972;29:117-121.

44. Sturgis E, Sacks PG, Masui H, Mendelsohn J, Schantz SP: Effects of antiepidermal growth factor receptor antibody 528 on the proliferation and differentiation of head and neck cancer. Otolaryngol Head Neck Surg 1994;111: 633-643.

45. Dudgeon D, Shinde SN, Hua $Y$, et al:: Implementation of a 220,000 Compound HCS campaign to identify disruptors of the interaction between p53 and hDM2, and characterization of the confirmed hits. J Biomol Screen 2010;15:152-174.

46. Johnston P, Foster CA, Shun TY, et al.: Development and implementation of a 384-well homogeneous fluorescence intensity high-throughput screening assay to identify mitogen-activated protein kinase phosphatase-1 dualspecificity protein phosphatase inhibitors. Assay Drug Dev Technol 2007;5: 319-332.

47. Johnston $\mathrm{P}$, Foster $\mathrm{CA}$, Tierno MB, et al:: Characterization of the $\mathrm{Cdc} 25 \mathrm{~B}$ dual specificity phosphatase inhibitor hits identified in a high throughput screen of the NIH compound library. Assays Drug Dev Technol 2009;7:250-265.

48. Dudgeon D, Shinde SN, Shun TY, et al:: Characterization and optimization of a novel protein-protein interaction biosensor HCS assay to identify disruptors of the interactions between p53 and hDM2. Assay Drug Dev Technol 2010;8: 437-458.

49. Johnston PA, Shinde SN, Hua Y, Shun TY, Lazo JS, Day BW: Development and validation of a high-content screening assay to identify inhibitors of cytoplasmic Dynein-mediated transport of glucocorticoid receptor to the nucleus. Assay Drug Dev Technol 2012;10:432-456.

50. Nickischer D, Laethem C, Trask OJ, Jr., et al.: Development and implementation of three mitogen-activated protein kinase (MAPK) signaling pathway imaging assays to provide MAPK module selectivity profiling for kinase inhibitors: MK2EGFP translocation, c-Jun, and ERK activation. Methods Enzymol 2006;414: 389-418.

51. Trask 0 , Nickischer D, Burton A, Williams RG, Kandasamy RA, Johnston PA, Johnston PA: High-throughput automated confocal microscopy imaging screen of a kinase-focused library to identify p38 mitogen-activated protein kinase inhibitors using the GE InCell 3000 analyzer. Methods Mol Biol 2009;565: 159-186.

52. Trask OJ, Jr., Baker A, Williams RG, et al:: Assay development and case history of a 32K-biased library high-content MK2-EGFP translocation screen to identify p38 mitogen-activated protein kinase inhibitors on the ArrayScan 3.1 imaging platform. Methods Enzymol 2006;414:419-439.

53. Williams RG, Kandasamy R, Nickischer D, et al.: Generation and characterization of a stable MK2-EGFP cell line and subsequent development of a high-content imaging assay on the Cellomics ArrayScan platform to screen for p38 mitogenactivated protein kinase inhibitors. Methods Enzymol 2006;414:364-389.

54. Chakravarti N, Myers JN, Aggarwal BB: Targeting constitutive and interleukin6-inducible signal transducers and activators of transcription 3 pathway in head and neck squamous cell carcinoma cells by curcumin (diferuloylmethane). Int J Cancer 2006;119:1268-1275.

55. Chen $\mathrm{C}$, Chen WC, Lu CH, et al:: Significance of interleukin- 6 signaling in the resistance of pharyngeal cancer to irradiation and the epidermal growth factor receptor inhibitor. Int J Radiat Oncol Biol Phys 2010;76:1214-1224.

56. Gasche J, Hoffmann J, Boland CR, Goel A: Interleukin-6 promotes tumorigenesis by altering DNA methylation in oral cancer cells. Int J Cancer 2011;129:10531063.

57. Shinriki S, Jono H, Ota K, et al:: Humanized anti-interleukin-6 receptor antibody suppresses tumor angiogenesis and in vivo growth of human oral squamous cell carcinoma. Clin Cancer Res 2009;15:5426-5434.

58. Su Y, Xie TX, Sano D, Myers JN: IL-6 stabilizes Twist and enhances tumor cell motility in head and neck cancer cells through activation of casein kinase 2 . PLoS One 2011;6:e19412.

59. Van Tubergen $E$, Vander Broek $R$, Lee J, et al.: Tristetraprolin regulates interleukin-6, which is correlated with tumor progression in patients with head and neck squamous cell carcinoma. Cancer 2011;117:2677-2689.

60. Yadav A, Kumar B, Datta J, Teknos TN, Kumar P: IL-6 promotes head and neck tumor metastasis by inducing epithelial-mesenchymal transition via the JAKSTAT3-SNAIL signaling pathway. Mol Cancer Res 2011;9:1658-1667.

61. Regis G, Pensa S, Boselli D, Novelli F, Poli V: Ups and downs: the STAT1:STAT3 seesaw of Interferon and gp130 receptor signalling. Semin Cell Dev Biol 2008; 19:351-359.

62. Lui VW, Boehm AL, Koppikar P, et al:: Antiproliferative mechanisms of a transcription factor decoy targeting signal transducer and activator of transcription (STAT) 3: the role of STAT1. Mol Pharmacol 2007;71:1435-1443.

63. Murray P: The JAK-STAT signaling pathway: input and output integration. J Immunol 2007;178:2623-2629.

64. Baell J, Holloway GA: New substructure filters for removal of pan assay interference compounds (PAINS) from screening libraries and for their exclusion in bioassays. J Med Chem 2010;53:2719-2740.

65. Walters W, Namchuk M: Designing screens: how to make your hits a hit. Nat Rev Drug Discov 2003;2:259-266.

66. Li $H_{1}$ Xiao $H_{\text {, Lin }} L$, et al:: Drug design targeting protein-protein interactions (PPIs) using multiple ligand simultaneous docking (MLSD) and drug repositioning: discovery of raloxifene and bazedoxifene as novel inhibitors of IL-6/GP130 interface. J Med Chem 2014;57:632-641. 


\section{JOHNSTON ET AL.}

67. Kessenbrock K, Plaks V, Werb Z: Matrix metalloproteinases: regulators of the tumor microenvironment. Cell 2010;141:52-67.

68. Mason S, Joyce JA: Proteolytic networks in cancer. Trends Cell Biol 2011;21: 228-237.

69. Rosenthal E, Matrisian LM: Matrix metalloproteases in head and neck cancer. Head Neck 2006;28:639-648.

70. LaPorte M, da Paz Lima DJ, Zhang F, et al.: 2-Guanidinoquinazolines as new inhibitors of the STAT3 pathway. Bioorg Med Chem Lett 2014;24:50815085.

\section{Abbreviations Used}

Ch1 $=$ fluorescent channel 1

$\mathrm{Ch} 2=$ fluorescent channel 2

$\mathrm{CM}=$ conditioned medium

DMEM = Dulbecco's modified Eagle's medium

DMSO $=$ dimethylsulfoxide

EGFR $=$ epidermal growth factor receptor

$\mathrm{EMT}=$ epithelial-mesenchymal transition

$F B S=$ fetal bovine serum

$\mathrm{FDA}=$ Food and Drug Administration

$\mathrm{HCS}=$ high-content screening

HNSCC $=$ head and neck squamous cell carcinoma

$I_{50}=50 \%$ inhibition concentration

IFN $\gamma=$ Interferon-gamma

IL-6 $=$ Interleukin-6

$\mathrm{IXU}=$ ImageXpress UItra

JAK $=$ Janus Kinase

mAbs $=$ monoclonal antibodies

MLSCN = Molecular Library Screening Center Network

$\mathrm{MMP}=$ matrix metalloprotease

$\mathrm{NCl}=$ National Cancer Institute

$\mathrm{NIH}=$ National Institutes of Health

$\mathrm{PBS}=$ phosphate-buffered saline

PMT $=$ Photomultiplier tube

pSTAT3-Y701 or pSTAT1 $=$ STAT1 phosphorylated at tyrosine 701

pSTAT3-Y705 or pSTAT3 $=$ STAT3 phosphorylated at tyrosine 705

pY $=$ phosphotyrosine

RTK = receptor tyrosine kinase

$\mathrm{S}: \mathrm{B}=$ signal-to-background

SFK $=$ Src family kinase

SFM $=$ serum-free medium

$\mathrm{SH} 2=$ Src homology 2 domain

SMR $=$ small-molecule repository

STAT1 = signal transducer and activator of transcription 1

STAT3 = signal transducer and activator of transcription 3

$\mathrm{TE}=$ translocation enhanced

TGF $\alpha=$ transforming growth factor alpha 